\title{
The 2003-2004 multisite photometric campaign for the $\beta$ Cephei and eclipsing star 16 (EN) Lacertae with an appendix on 2 Andromedae, the variable comparison star
}

\author{
M. Jerzykiewicz, ${ }^{1 \star}$ G. Handler, ${ }^{2}$ J. Daszyńska-Daszkiewicz, ${ }^{1}$ A. Pigulski, ${ }^{1}$ E. Poretti, ${ }^{3}$ \\ E. Rodríguez, ${ }^{4}$ P. J. Amado, ${ }^{4}$ Z. Kołaczkowski, ${ }^{1}$ K. Uytterhoeven, ${ }^{5,6}$ \\ T. N. Dorokhova, ${ }^{7}$ N. I. Dorokhov, ${ }^{7}$ D. Lorenz,${ }^{8}$ D. Zsuffa, ${ }^{9}$ S.-L. Kim, ${ }^{10}$ \\ P.-O. Bourge, ${ }^{11}$ B. Acke, ${ }^{5}$ J. De Ridder, ${ }^{5}$ T. Verhoelst, ${ }^{5}$ R. Drummond, ${ }^{5}$
} A. I. Movchan, ${ }^{7}$ J.-A. Lee, ${ }^{10}$ M. Stęślicki, ${ }^{12}$ J. Molenda-Żakowicz, ${ }^{1}$ R. Garrido, ${ }^{4}$ S.-H. Kim, ${ }^{10}$ G. Michalska, ${ }^{1}$ M. Paparó, ${ }^{9}$ V. Antoci ${ }^{8}$ and C. Aerts ${ }^{5}$

${ }^{1}$ Astronomical Institute of the Wroctaw Univeristy, Kopernika 11, PL-51-622 Wrocław, Poland

${ }^{2}$ Copernicus Astronomical Center, Bartycka 18, PL-00-716 Warsaw, Poland

${ }^{3}$ INAF-Osservatorio Astronomico di Brera, Via Bianchi 46, I-23807 Merate, Italy

${ }^{4}$ Instituto de Astrofisica de Andalucia, C.S.I.C., Apdo. 3004, E-18080 Granada, Spain

${ }^{5}$ Instituut voor Sterrenkunde, K. U. Leuven, Celestijnenlaan 200B, B-3001 Leuven, Belgium

${ }^{6}$ Mercator Telescope, Calle Alvarez de Abreu 70, E-38700 Santa Cruz de La Palma, Spain

${ }^{7}$ Astronomical Observatory of Odessa National University, Marazlievskaya, 1v, UA-65014 Odessa, Ukraine

${ }^{8}$ Institut für Astronomie, Universität Wien, Türkenschanzstrasse 17, A-1180 Wien, Austria

${ }^{9}$ Konkoly Observatory, MTA CSFK, Konkoly Thege Miklós út 15-17., 1121 Budapest, Hungary

${ }^{10}$ Korea Astronomy and Space Science Institute, Daejeon 305-348, Korea

${ }^{11}$ Institut d'Astrophysique et de Géophysique, Université de Liège, allée du Six Août 17, B-4000 Liège, Belgium

${ }^{12}$ Space Research Centre, Polish Academy of Sciences, Solar Physics Division, ul. Kopernika 11, PL-51-622 Wroctaw, Poland

Accepted 2015 August 21. Received 2015 August 20; in original form 2015 February 3

\begin{abstract}
A multisite photometric campaign for the $\beta$ Cephei and eclipsing variable 16 Lacertae is reported. $749 \mathrm{~h}$ of high-quality differential photoelectric Strömgren, Johnson and Geneva time series photometry were obtained with 10 telescopes during 185 nights. After removing the pulsation contribution, an attempt was made to solve the resulting eclipse light curve by means of the computer program EBOP. Although a unique solution was not obtained, the range of solutions could be constrained by comparing computed positions of the secondary component in the Hertzsprung-Russell diagram with evolutionary tracks. For three highamplitude pulsation modes, the $u v y$ and the Geneva $U B G$ amplitude ratios are derived and compared with the theoretical ones for spherical-harmonic degrees $\ell \leq 4$. The highest degree, $\ell=4$, is shown to be incompatible with the observations. One mode is found to be radial, one is $\ell=1$, while in the remaining case $\ell=2$ or 3 . The present multisite observations are combined with the archival photometry in order to investigate the long-term variation of the amplitudes and phases of the three high-amplitude pulsation modes. The radial mode shows a non-sinusoidal variation on a time-scale of $73 \mathrm{yr}$. The $\ell=1$ mode is a triplet with unequal frequency spacing, giving rise to two beat-periods, $720.7 \mathrm{~d}$ and $29.1 \mathrm{yr}$. The amplitude and phase of the $\ell=2$ or 3 mode vary on time-scales of $380.5 \mathrm{~d}$ and $43 \mathrm{yr}$. The light variation of 2 And, one of the comparison stars, is discussed in the appendix.
\end{abstract}

Key words: stars: early-type-stars: individual: 16 (EN) Lacertae-stars: individual: 2 Andromedae-stars: oscillations.

^E-mail: jerzykiewicz@astro.uni.wroc.pl 


\section{INTRODUCTION}

16 (EN) Lacertae $=$ HR 8725 (B2 IV, $V=5.59)$, a member of Lac OB1a, is a single-lined spectroscopic binary (Lee 1910; Struve \& Bobrovnikoff 1925) and an eclipsing variable. The orbital period, derived from the epochs of minimum light, $P_{\text {orb }}=12.09684 \mathrm{~d}$ (Jerzykiewicz 1980; Pigulski \& Jerzykiewicz 1988). The system consists of a well-known $\beta$ Cephei variable and an undetected secondary. The $\beta$ Cephei variation of the primary is dominated by three pulsation modes with frequencies close to $6 \mathrm{~d}^{-1}$. According to Fitch (1969, henceforth F69), who based his analysis on archival 1951, 1952 and 1954 radial-velocity (RV) and photoelectric blue-light observations, the first two modes have constant amplitudes, while the amplitude of the third mode varies on a time-scale of months. F69 described the first two modes as singlets, having frequencies $f_{1}=5.91134$ and $f_{2}=5.85286 \mathrm{~d}^{-1}$, and the third mode as a doublet, consisting of two terms with frequencies $f_{3,1}=5.49990$ and $f_{3,2}=f_{1}$ $-5 f_{\text {orb }}=5.49799 \mathrm{~d}^{-1}$. Comparable frequencies were derived from the 1964 and 1965 photoelectric observations, obtained by one of us (MJ) at Lowell Observatory, viz. $f_{1}=5.91120 \pm 0.00005$, $f_{2}=5.85503 \pm 0.00010$, and $f_{3}=5.50322 \pm 0.00009 \mathrm{~d}^{-1}$ (Jarzȩbowski et al. 1979). The first value agrees with that of F69, but the other two differ from F69's $f_{2}$ and $f_{3,1}$ by 0.0022 and $0.0033 \mathrm{~d}^{-1}$, respectively. From these differences, Jarzȩbowski et al. (1979) concluded that F69's values of $f_{2}$ and $f_{3,1}$ suffered from an error of one cycle per year $\left(\mathrm{yr}^{-1}\right)$.

An analysis of all photometric observations of 16 Lac obtained throughout 1992 was carried out by Jerzykiewicz \& Pigulski (1996, 1999, henceforth JP96, JP99). The main results of the analysis can be summarized as follows: (1) the amplitudes of the $f_{1}$ and $f_{2}$ modes vary on a time-scale of decades, the reciprocal timescales amounting to 0.014 and $0.020 \mathrm{yr}^{-1}$, respectively, (2) the third mode is confirmed to be a doublet, but with frequencies different from those derived by F69, viz. $f_{3,1}=5.5025779 \pm 0.0000005$ and $f_{3,2}=5.5040531 \pm 0.0000008 \mathrm{~d}^{-1}$. Note that none of these frequencies bears a simple numerical relation to the orbital period. Moreover, JP96 demonstrated that there is no correlation of the pulsation amplitudes with the orbital phase.

The RV data available at the time were shown by JP96 to be consistent with the above-mentioned photometric results. The RV data, however, were much less numerous than the photometric data, making this conclusion somewhat uncertain. The situation has improved after Lehmann et al. (2001, henceforth L01), provided new RV observations, more than doubling the number of existing measurements. A periodogram analysis of these and the older data led L01 to a number of frequency solutions, the details of which depended on the weights assigned to the RV measurements from a particular source. As far as the periods are concerned, the results valid for all weighting schemes can be summarized as follows: (1) the first mode is an equidistant triplet with the central term having the largest amplitude and a period, $P_{1}$, equal to $0.16916707 \mathrm{~d}$ $\left(f_{1}=5.9113160 \mathrm{~d}^{-1}\right)$. The remaining two periods, $P_{1}^{+}$and $P_{1}^{-}$in the notation of L01, are equal to 0.16916605 and $0.16916809 \mathrm{~d}$, respectively. The frequency separation of the triplet amounts to $0.0000356 \mathrm{~d}^{-1}$, corresponding to the amplitude-modulation frequency of $0.0130 \mathrm{yr}^{-1},(2)$ the second mode is a doublet; in the order of decreasing amplitude, the periods, $P_{2}$ and $P_{2}^{+}$in the notation of L01, are equal to 0.17085553 and $0.17077074 \mathrm{~d}$, respectively $\left(f_{2}=5.8528981\right.$ and $\left.f_{2}^{+}=5.8558041 \mathrm{~d}^{-1}\right)$, resulting in a beat period of $344 \mathrm{~d}$ (the beat frequency of $0.00291 \mathrm{~d}^{-1}$ ), (3) the third mode is also a doublet; in the order of decreasing amplitude the periods are $P_{3}=0.18173251$ and $P_{3}^{+}=0.18168352 \mathrm{~d}\left(f_{3}=5.5025928\right.$ and $\left.f_{3}^{+}=5.5040765 \mathrm{~d}^{-1}\right)$; in this case the beat period is equal to $674 \mathrm{~d}$. Conclusions (1) and (3) approximately agree with the results of JP96 and JP99, but conclusion (2) does not. The disagreement is twofold. First, the time-scale of the amplitude modulation of the $f_{2}$ term derived by L01 is less than one fiftieth of that derived by JP96 and JP99. Secondly, L01's $f_{2}$, i.e. the frequency of the higher amplitude component of the $f_{2}, f_{2}^{+}$doublet has a value close to that originally determined by F69 and dismissed by Jarzȩbowski et al. (1979) as a yearly alias of the photometric $f_{2}$ value they derived. Note that $f_{2}$ of Jarzębowski et al. (1979) is close to $f_{2}^{+}$, the frequency of the smaller amplitude component of the $f_{2}, f_{2}^{+}$doublet. This, of course, is the consequence of the doublet's beat-period having its value close to $1 \mathrm{yr}$.

In addition to the three modes just discussed, six fainter terms were detected by Jerzykiewicz (1993) in his 1965 data. The $v$ amplitudes of these terms ranged from $2.1 \pm 0.14$ to $0.7 \pm 0.14 \mathrm{mmag}$, and the frequencies were equal to (in the order of decreasing amplitude) $0.1653,7.194,11.822,11.358,11.414$, and $11.766 \mathrm{~d}^{-1}$. The first of these is equal to twice the orbital frequency, suggesting ellipsoidal variability. However, the observed amplitude and phase excluded this possibility. The third frequency is equal to $2 f_{1}$ and the three last frequencies are the combination terms $f_{2}+f_{3}, f_{1}+f_{3}$ and $f_{1}+f_{2}$. The 7.194- $\mathrm{d}^{-1}$ term was attributed by Jerzykiewicz (1993) to an independent pulsation mode and was used as such, together with the three strongest ones, in an asteroseismic study of the star (Dziembowski \& Jerzykiewicz 1996). Subsequently, however, this term was shown by Sareyan et al. (1997) and Handler et al. (2006) to be due to a light variation of 2 Andromedae, used by Jerzykiewicz (1993) as a comparison star.

During a multisite photometric campaign carried out between 2003 August 2 and 2004 January 9, 16 Lac has been observed together with the $\beta$ Cephei variable 12 (DD) Lacertae. Results of the observations of $12 \mathrm{Lac}$ and their analysis were published some years ago (Handler et al. 2006). In the next section, we describe the 2003-2004 multisite campaign's photometric observations and reductions. In Section 3, we carry out a frequency analysis of the campaign's uvy time series of 16 Lac. In Section 4, we use the analysis results to remove the intrinsic component of the variation from the time series, thus bringing out the eclipse, and discuss the eclipse solutions and the evolutionary state of the secondary component. In Section 5, we derive the primary component's fundamental parameters. Section 6 is devoted to determining the harmonic degree of the three highest amplitude pulsation modes of 16 Lac. The long-term variation of the photometric amplitudes and phases of these three modes is investigated in Section 7 using the present and archival data. The last section contains a summary and discussion of the results. The light variation of 2 And is examined in the appendix.

\section{OBSERVATIONS AND REDUCTIONS}

Our 2003-2004 photometric observations of 16 Lac were carried out at 10 observatories on three continents with small- to medium-sized telescopes (see Table 1). In most cases, single-channel differential photoelectric photometry was acquired. At the Sobaeksan Optical Astronomy Observatory (SOAO) and the Białków Observatory (BO) the photometry was done with CCD cameras. Wherever possible, Strömgren $u v y$ filters were used. At the Sierra Nevada and San Pedro Martir (SPM) Observatories simultaneous $u v b y$ photometers were available, including $b$ filters as well. However, the $u$ data from SPM were unusable. At BO, a Strömgren $y$ filter was used. At four observatories where no Strömgren filters were available, we used 
Table 1. Log of the photometric measurements of 16 Lac. Observatories are listed in the order of their geographic longitude.

\begin{tabular}{|c|c|c|c|c|c|c|c|}
\hline \multirow[b]{2}{*}{ Observatory } & \multirow[b]{2}{*}{ Longitude } & \multirow[b]{2}{*}{ Latitude } & \multirow[b]{2}{*}{ Telescope } & \multicolumn{2}{|c|}{ Amount of data } & \multirow[b]{2}{*}{ Filter(s) } & \multirow[b]{2}{*}{ Observer(s) } \\
\hline & & & & (Nights) & (h) & & \\
\hline Sierra Nevada & $-3^{\circ} 23^{\prime}$ & $37^{\circ} 04^{\prime}$ & $0.9 \mathrm{~m}$ & 18 & 75.9 & uvby & ER, PJA, RG \\
\hline Mercator & $-17^{\circ} 53^{\prime}$ & $28^{\circ} 46^{\prime}$ & $1.2 \mathrm{~m}$ & 33 & 104.2 & Geneva & $\begin{array}{l}\text { KU, RD, JDD, TV, } \\
\text { JDR, BA, POB }\end{array}$ \\
\hline Fairborn & $-110^{\circ} 42^{\prime}$ & $31^{\circ} 23^{\prime}$ & 0.75-m APT & 57 & 205.5 & uvy & - \\
\hline Lowell & $-111^{\circ} 40^{\prime}$ & $35^{\circ} 12^{\prime}$ & $0.5 \mathrm{~m}$ & 19 & 96.8 & uvy & MJ \\
\hline SPM & $-115^{\circ} 28^{\prime}$ & $31^{\circ} 03^{\prime}$ & $1.5 \mathrm{~m}$ & 10 & 36.4 & uvby & EP, JPS, LP \\
\hline $\begin{array}{l}\text { Sobaeksan Optical } \\
\text { Astronomy }\end{array}$ & $+128^{\circ} 27^{\prime}$ & $36^{\circ} 56^{\prime}$ & $0.6 \mathrm{~m}$ & 5 & 31.9 & $V$ & SLK, JAL, SHK \\
\hline Mt. Dushak-Erekdag & $+57^{\circ} 53^{\prime}$ & $37^{\circ} 55^{\prime}$ & $0.8 \mathrm{~m}$ & 12 & 63.4 & V & TND, NID \\
\hline Mayaki & $+30^{\circ} 17^{\prime}$ & $46^{\circ} 24^{\prime}$ & $0.5 \mathrm{~m}$ & 6 & 13.0 & $V$ & AIM \\
\hline Piszkéstető & $+19^{\circ} 54^{\prime}$ & $47^{\circ} 55^{\prime}$ & $0.5 \mathrm{~m}$ & 13 & 56.1 & $V$ & MP, DZ, DL, VA \\
\hline Białków & $+16^{\circ} 40^{\prime}$ & $51^{\circ} 29^{\prime}$ & $0.6 \mathrm{~m}$ & 12 & 67.2 & $y$ & ZK, GM, JMŻ, AP, MS \\
\hline Total & & & & 185 & 749.3 & & \\
\hline
\end{tabular}

Johnson's $V$. Finally, as the photometer at the Mercator telescope had Geneva filters installed permanently, we used this filter system. We chose the two 'classical' comparison stars: $10 \mathrm{Lac}(\mathrm{O} 9 \mathrm{~V}$, $V=4.88$ ) and 2 And (A3Vn, $V=5.09$ ). A check star, HR 8708 $(\mathrm{A} 3 \mathrm{Vm}+\mathrm{F} 6 \mathrm{~V}, V=5.81)$, was additionally observed during one of the SPM runs. HD 216854 (F5, $V=7.31$ ) was used as a comparison star at SOAO; its constancy was checked against two fainter stars. At $\mathrm{BO}, \mathrm{BD}+40^{\circ} 4950 \mathrm{a}=\mathrm{PPM} 63607=\mathrm{GSC} 3223-01835(\mathrm{~F} 5 / \mathrm{K} 0$, $V=9.29)$ was used as a sole comparison star. In order to compensate for the large brightness difference between the programme and the comparison star $(\sim 3.7 \mathrm{mag})$, BO observers took a sequence of CCD frames with short and long exposure-times, such that 16 Lac was well exposed on the frames with short exposure-times, while the comparison star, on the frames with long exposure-times. Reductions of the SOAO and BO observations included calibrating all CCD frames in a standard way and processing them with the DAOPHOT II package (Stetson 1987). Then, magnitudes were obtained by means of aperture photometry, the magnitudes of the comparison stars were interpolated for the times of observation of $16 \mathrm{Lac}$ and differential magnitudes were calculated. In the case of the BO data, several consecutive data points were averaged, resulting in the final time series.

As we mentioned in the Introduction, 2 And turned out to be a low-amplitude periodic variable. We shall discuss the variability of 2 And in the appendix. No evidence for photometric variability of $10 \mathrm{Lac}$ was found. We thus proceeded by pre-whitening the variability of 2 And with a fit determined from all its differential magnitudes relative to $10 \mathrm{Lac}$. The residual magnitudes of 2 And were then combined with the 10 Lac data into a curve that was assumed to reflect the effects of transparency and detector sensitivity changes only. These combined time series were binned into intervals that would allow good compensation for the above-mentioned nonintrinsic variations in the target star time series and were subtracted from the measurements of 12 and 16 Lac. Note that the binning minimizes the noise in the differential light curves of the targets. Finally, the photometric zero-points of the different instruments were compared and adjusted if required. In particular, adjustments were necessary for the SOAO and BO observations because they were obtained with different comparison stars. Measurements in the Strömgren $y$ and the Johnson and Geneva $V$ filters were analysed together because these filters have very nearly the same effective wavelength; the combined $y, V$ light curve is henceforth referred to as 'the $y$ data.' For further details of the reductions, common to 12 and 16 Lac, see Handler et al. (2006).
For both stars, 12 and $16 \mathrm{Lac}$, the $y$ data were the most extensive by far. In the case of 16 Lac, there were $3190 y$-filter measurements (including Johnson and Geneva $V$-filter measurements, see above), $2012 v$-filter measurements, and $1686 u$-filter measurements. The Mercator, Fairborn, Lowell, and Mayaki data included measurements falling between the first and the fourth contacts of six eclipses. Omitting these measurements resulted in the time series suitable for frequency analysis; in the process, the number of measurements was reduced to 3055,1895 , and 1583 in $y, v$, and $u$, respectively. In all cases, the measurements spanned an interval of $179.2 \mathrm{~d}$. The number of measurements in the Geneva filters was 411 , with 395 taken outside eclipses. In both cases the data spanned 129.9 d. A sample of the light curves of 16 Lac is presented in Fig. 1.

\section{FREQUENCY ANALYSIS}

Using the results of JP99's analysis (see the Introduction), we can predict blue-light amplitudes of the $f_{i}(i=1,2,3)$ modes for the epochs of our 2003-2004 observations. In the case of $f_{1}$ and $f_{2}$, the amplitudes predicted under an assumption of sinusoidal variations with the reciprocal time-scales of 0.014 and $0.020 \mathrm{yr}^{-1}$ amount to 12.4 and $15.1 \mathrm{mmag}$, respectively, and both amplitudes should be very nearly constant over the 179.2-d interval spanned by the data. In the case of $f_{3}$, the amplitude should increase from 3.9 to 9.6 mmag over this interval. A preliminary analysis of the $y$ data showed that these predictions are in error, grossly so in the case of the first two modes. In order to examine this issue in detail, we divided the $y$, $v$, and $u$ data into adjacent segments; except for the first segments in all filters and the last two segments in $v$ and $u$, the segments partly overlapped. The first $y$ segment spanned $40 \mathrm{~d}$ and each of the remaining $y, v$, and $u$ segments, about $29 \mathrm{~d}$. In each segment, we then derived the amplitudes, $A_{i}(i=1,2,3)$, by the method of least squares using the following observational equations:

$\Delta m_{j}=A_{0}+\sum_{i=1}^{N} A_{i} \cos \left(2 \pi f_{i} t_{j}+\Phi_{i}\right)$,

where $N=3, \Delta m_{j}$ are the $y, v$, or $u$ differential magnitudes, $f_{i}(i=1$, $2,3)$ are assumed to be equal to $5.9112,5.8550$ and $5.5032 \mathrm{~d}^{-1}$, respectively, and $t_{j}$ are HJD 'minus' an arbitrary initial epoch. The assumed values of the frequencies $f_{i}(i=1,2,3)$ are approximately equal to the weighted means of the values given in Tables 4,5 and 8 , respectively, with the weights equal to the corresponding amplitudes 


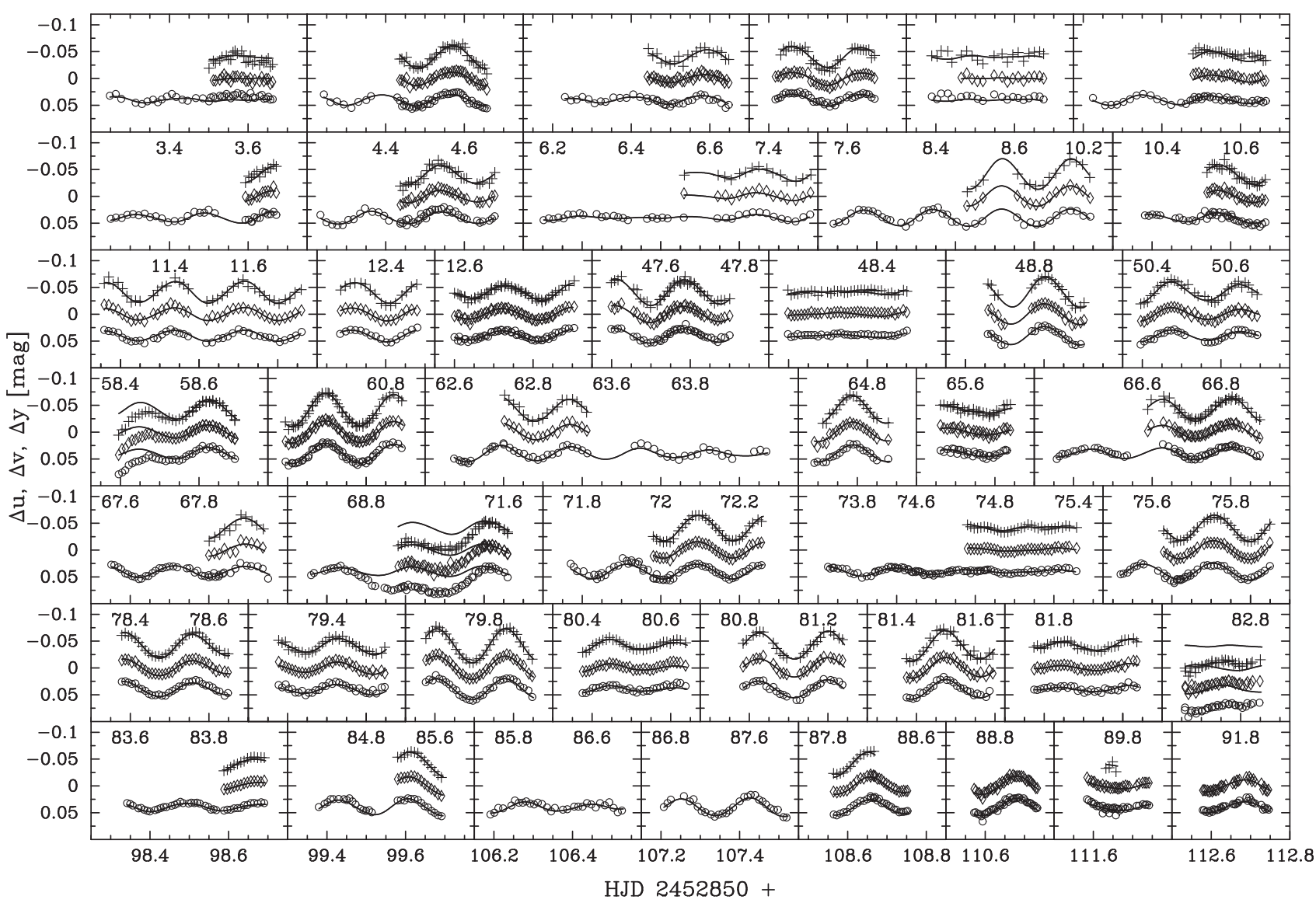

Figure 1. A sample of our $u, v$, and $y$ light curves of 16 Lac (plus signs, diamonds, and circles, respectively). The lines are the synthetic light curves computed as explained in Section 3. Note the deviation of the measurements from the fit due to three consecutive eclipses on the nights JD 2452917 , JD 2452929 , and JD 245 2941. The amount of data displayed is about half the total.

(see Section 7). Note that the first $y$ segment covers about two cycles of the longest beat-period in the variation of $16 \mathrm{Lac}$, the one arising from the interference of $f_{1}$ and $f_{2}$, and the remaining segments, about 1.5 cycle. An examination of the residuals from the leastsquares solutions revealed six deviant observations in the second $y$ segment, all from the Mayaki Observatory. After rejecting these observations, the residuals in all but one segment fell within an interval of $\pm 0.015 \mathrm{mag}$; the exception was the first $u$ segment in which there were five residuals outside the \pm 0.015 mag interval, but all smaller in their absolute value than $0.018 \mathrm{mag}$.

Results of the above exercise are presented in Fig. 2 for the $y$ and $v$ amplitudes (points and circles, respectively), and in Fig. 3 for $u$. The error bars shown in the figures extend one standard deviation on each side of the plotted symbols. However, the standard deviations are not the formal standard deviations of the least-squares solutions of equations (1) but twice the formal standard deviations. In this, we follow Handler et al. (2000) and Jerzykiewicz et al. (2005) who - while dealing with time series observations similar to the present ones - showed that the formal standard deviations were underestimated by a factor of about 2 .

The solid line in the bottom panel of Fig. 2 shows the predicted blue-light amplitude of the $f_{3}$ mode (see the first paragraph of this section). The predicted blue-light amplitude of the $f_{1}$ mode, $12.4 \mathrm{mmag}$, is a factor of about 2 greater than the observed $y$ and $v$ amplitudes seen in the top panel of Fig. 2. In the case of the $f_{2}$ mode, the prediction fails on two accounts: (1) the observed amplitudes vary from nearly zero to about 0.8 of the predicted value of

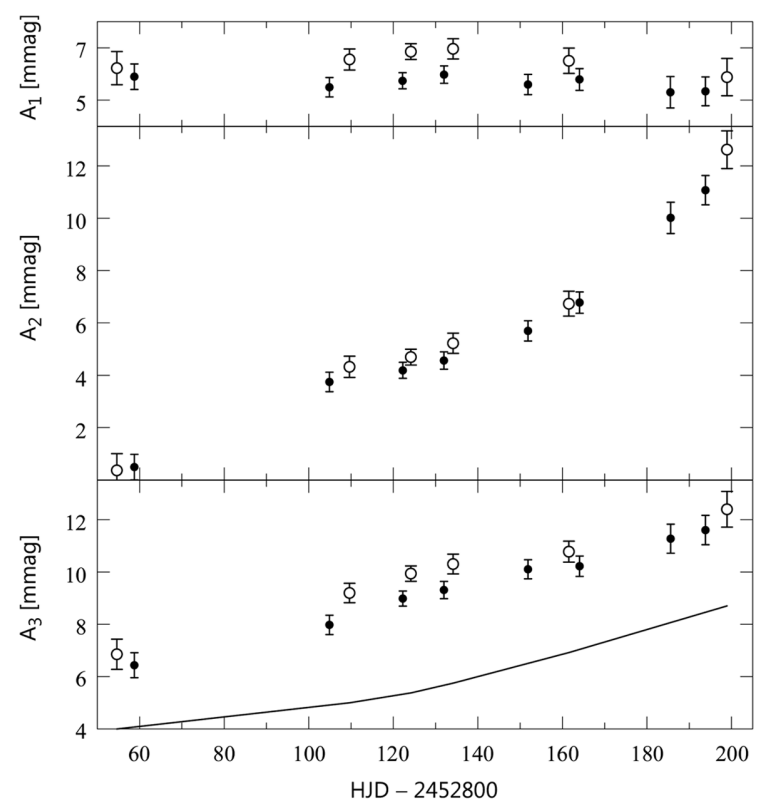

Figure 2. The $y$ (points) and $v$ (circles) amplitudes of the $f_{1}, f_{2}$, and $f_{3}$ modes of $16 \mathrm{Lac}$ (from top to bottom) plotted as a function of HJD. The line in the bottom panel shows predicted blue-light amplitude of the $f_{3}$ mode (see the text for details). 


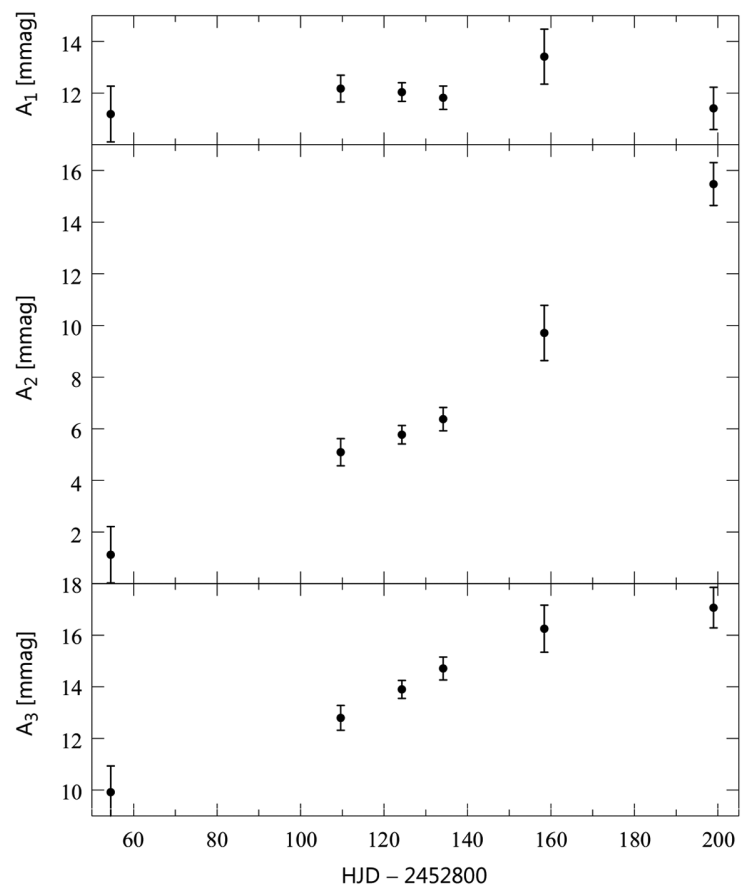

Figure 3. The $u$ amplitudes of the $f_{1}, f_{2}$, and $f_{3}$ modes of 16 Lac (from top to bottom) plotted as a function of HJD.

$15.1 \mathrm{mmag}$ on a time-scale about 50 times shorter than that determined by JP99, and (2) the median values of the observed $y$ and $v$ amplitudes are a factor of about 2 smaller than predicted blue-light amplitude.

Standard frequency analysis and successive pre-whitening with sinusoids applied to the 2003-2004 y magnitudes yielded the following frequencies (in the order they were identified from the power spectra): $5.503,5.591,5.856$ and $5.852 \mathrm{~d}^{-1}$. The first three numbers are very nearly equal to $f_{3}, f_{1}$ and $f_{2}$, while the fourth is close to $f_{2}$. The amplitudes amounted to 8.8, 5.9, 4.3 and $1.8 \mathrm{mmag}$, respectively. The first two amplitudes are close to the mean $y$ amplitudes $A_{3}$ and $A_{1}$, while the sum of the third and the fourth is close to the mean $A_{2}$ (see Fig. 2). In addition, the fourth frequency differs from the third by less than the frequency resolution of the data. Clearly, the fourth frequency is an artefact. This shows that because of the variable amplitudes of the $f_{2}$ and $f_{3}$ modes, the usual procedure of pre-whitening with sinusoids is inadequate in the present case. Therefore, instead of the usual procedure, we tried the following two methods: (1) pre-whitening separately in each segment, (2) pre-whitening with $A_{1}$ assumed constant, $A_{2}$ assumed to vary quadratically with time, and $A_{3}$, linearly. Both methods were applied to the $y$ data; in the case of $v$ and $u$, we limited ourselves to method 1 . In method 1 , we computed residuals from the leastsquares fit of equation (1) in each segment. Then, we took straight means of the residuals for a given $t_{j}$ in the overlapping parts of adjacent segments, so that for each $t_{j}$ there was one residual. In method 2, we used equation (1) with $A_{1}=$ const, $A_{2}=B_{1}+C_{1} t_{j}+D_{1} t_{j}^{2}$, and $A_{3}=B_{2}+C_{2} t_{j}$. Since the first segment's $A_{2}$ would not fit the quadratic relation derived from the remaining segments (see the middle panel of Fig. 2), we applied the quadratic equation separately to this segment and then to the remaining data. In this case, there was no need to average residuals because the first and second segment do not overlap. Using the residuals as data, we then computed the amplitude spectra and the signal-to-noise ratio $(\mathrm{S} / \mathrm{N})$ as a function of frequency, where $S$ is the amplitude for a given

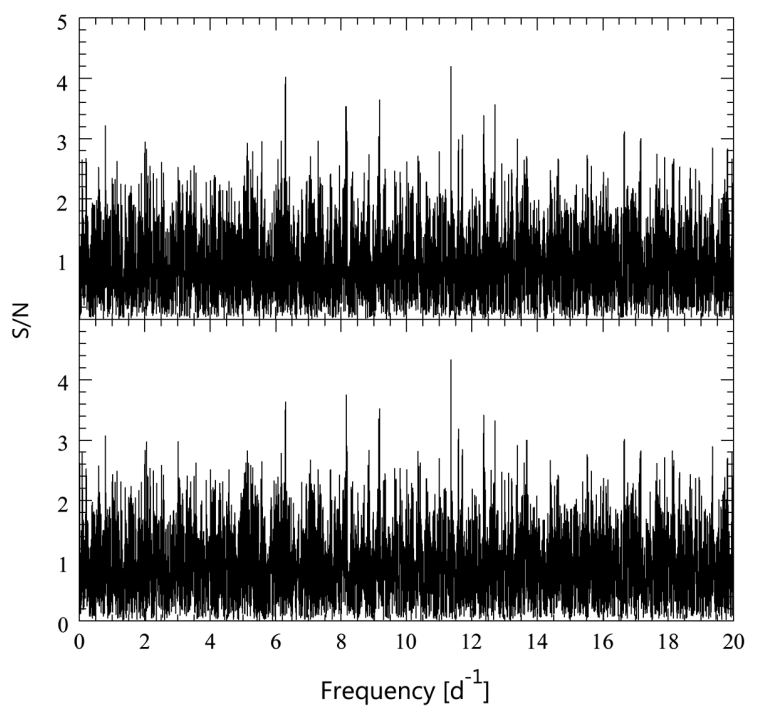

Figure 4. The $\mathrm{S} / \mathrm{N}$ spectra, computed from the $y$ data pre-whitened with the frequencies $f_{1}=5.9112 \mathrm{~d}^{-1}, f_{2}=5.8550 \mathrm{~d}^{-1}$, and $f_{3}=5.5032 \mathrm{~d}^{-1}$ by means of method 1 (upper panel) and method 2 (lower panel). For details, see the text.

frequency, and $N$ is the mean amplitude in $1 \mathrm{~d}^{-1}$ frequency intervals; in the first frequency interval, we omitted the amplitudes for $f \leq 1 / T$, where $T$ is the total span of the data. For the $y$ residuals, the $\mathrm{S} / \mathrm{N}$ are plotted in Fig. 4 as a function of frequency. We shall refer to the plots of this sort as the signal-to-noise spectra or $\mathrm{S} / \mathrm{N}$ spectra. In Fig. 4, the S/N spectra are shown for method 1 and 2 in the upper and lower panel, respectively. In both cases, the highest $\mathrm{S} / \mathrm{N}$ peak occurs at $11.359 \mathrm{~d}^{-1}$, a frequency very nearly equal to the combination frequency $f_{2}+f_{3}$. The corresponding amplitudes amount to 0.56 and $0.59 \mathrm{mmag}$, and the $\mathrm{S} / \mathrm{N}$ values are equal to 4.3 and 4.5 , respectively. Thus, both peaks are significant according to the popular criterion of Breger et al. (1993). In the $\mathrm{S} / \mathrm{N}$ spectrum of method 1 residuals pre-whitened with $f_{2}+f_{3}$, the highest peak, having the amplitude of $0.64 \mathrm{mmag}$ and $\mathrm{S} / \mathrm{N}=4.2$, occurred at $6.299 \mathrm{~d}^{-1}$. In the analogous spectrum of method 2 residuals, there were no peaks with $\mathrm{S} / \mathrm{N}>4$; the peak at $6.299 \mathrm{~d}^{-1}$ had $\mathrm{S} / \mathrm{N}=3.4$. The frequency of $6.299 \mathrm{~d}^{-1}$ we shall refer to as $f_{4}$. In the $\mathrm{S} / \mathrm{N}$ spectrum of method 1 residuals pre-whitened with the five frequencies, there was a peak at $0.085 \mathrm{~d}^{-1}$, rather close to $f_{\text {orb }}$, but there was no peak at $2 f_{\text {orb }}$, although one was present at this frequency in the power spectra of the $1965 \mathrm{~V}$ data (see fig. 1 of Jerzykiewicz 1993). The amplitude and $\mathrm{S} / \mathrm{N}$ at $f_{\text {orb }}$ were equal to $0.55 \mathrm{mmag}$ and 2.1, respectively. At $2 f_{\text {orb }}$, the corresponding numbers were $0.34 \mathrm{mmag}$ and 1.3 , respectively; the $1965 \mathrm{~V}$ amplitude was equal to $2.1 \mathrm{mmag}$.

In the case of the $v$-filter residuals, computed using method 1 , the $\mathrm{S} / \mathrm{N}$ spectrum showed the highest peak at $6.301 \mathrm{~d}^{-1}$, very nearly equal to $f_{4}$. In the second $\mathrm{S} / \mathrm{N}$ spectrum, obtained from the residuals computed with this frequency included in pre-whitening, the highest peak occurred at $11.359 \mathrm{~d}^{-1}$, the same combination frequency as that found in the $y$ residuals. The $\mathrm{S} / \mathrm{N}$ amounted to 3.8 and 3.9 in the first and the second spectrum, so that in the second spectrum the Breger et al. (1993) criterion of $\mathrm{S} / \mathrm{N}>3.5$ for a combination frequency was satisfied. The amplitudes were now greater than the $y$-filter ones, viz. 0.61 and $0.64 \mathrm{mmag}$, respectively. At low frequencies, there were peaks close to $f_{\text {orb }}$ and $2 f_{\text {orb }}$. The $\mathrm{S} / \mathrm{N}$ and the amplitude at $f_{\text {orb }}$ amounted to 2.7 and $0.66 \mathrm{mmag}$, while at $2 f_{\text {orb }}$, to 2.4 and $0.58 \mathrm{mmag}$. The phase of maximum light of the $f_{\text {orb }}$ term was $0.49 \pm 0.04$ orbital phase, suggesting a reflection effect. In the 


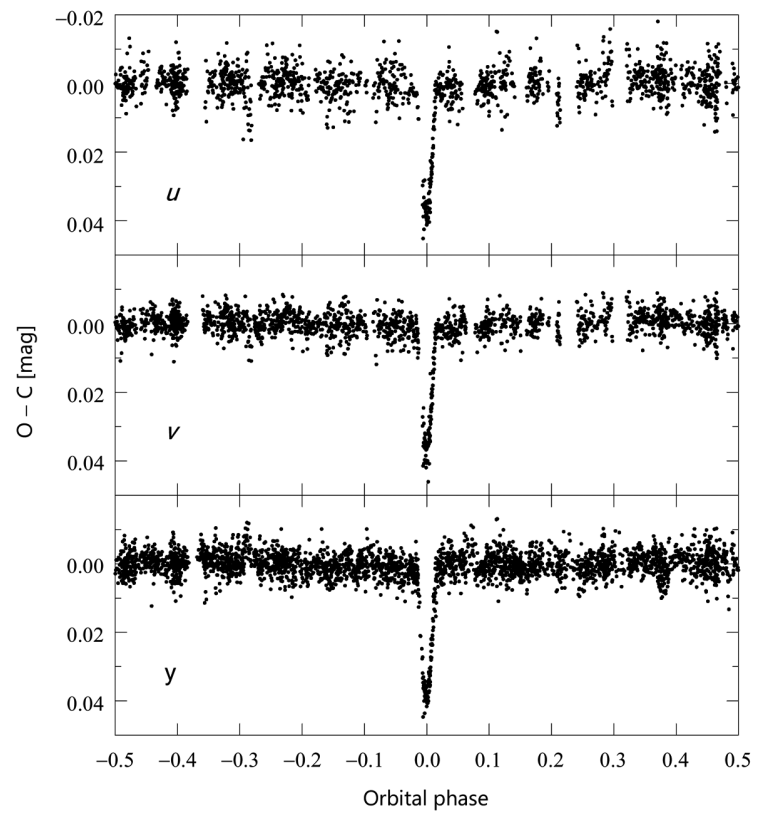

Figure 5. The $y, v$ and $u$ residuals from the five-term pulsational solutions plotted as a function of orbital phase. The $y$ deviant points (see Fig. 6) are not shown.

case of the $2 f_{\text {orb }}$ term, the phase of maximum light was $0.51 \pm 0.04$ orbital phase, excluding an ellipsoidal variation as the cause. The $1965 \mathrm{~B}$ amplitude at $2 f_{\text {orb }}$ was equal to $1.6 \mathrm{mmag}$, and the phase of maximum light was $0.68 \pm 0.02$ orbital phase (see Jerzykiewicz 1993, table 7). In the case of the $u$-filter residuals, the highest peak in the first $\mathrm{S} / \mathrm{N}$ spectrum was at $12.359 \mathrm{~d}^{-1}$, the $+1 \mathrm{~d}^{-1}$ alias of the combination frequency $f_{2}+f_{3}$. In the second $\mathrm{S} / \mathrm{N}$ spectrum, the highest peak occurred at $1.092 \mathrm{~d}^{-1}$, and the second highest peak, at $6.301 \mathrm{~d}^{-1}$. At $11.359 \mathrm{~d}^{-1}$ in the first spectrum and at $6.301 \mathrm{~d}^{-1}$ in the second spectrum, $\mathrm{S} / \mathrm{N}$ were equal to 3.9 and 3.1 , respectively, and the $f_{2}+f_{3}$ and $f_{4}$ amplitudes were equal to 0.82 and $0.80 \mathrm{mmag}$. At $f_{\text {orb }}$, the $\mathrm{S} / \mathrm{N}$ and the amplitude amounted to 2.0 and $0.75 \mathrm{mmag}$, while at $2 f_{\text {orb }}$, to 1.0 and 0.38 mmag.

The $\mathrm{S} / \mathrm{N}$ spectrum of the $y$ residuals, computed by means of method 1 but with all five significant terms (i.e. $f_{1}, f_{2}, f_{3}, f_{2}+f_{3}$, and $f_{4}$ ) included showed no peaks higher than 3.8. We decided to terminate the frequency analysis at this stage. The $y, v$, and $u$ fits computed with the five terms taken into account were used to plot the synthetic light curves in Fig. 1.

\section{THE ECLIPSE}

\subsection{The EBOP solutions}

The residuals, computed by means of method 1 with the five terms taken into account (see the last paragraph of the preceding section) for all $y, v$, and $u$ observations, including those obtained during eclipses, are plotted in Fig. 5 as a function of orbital phase. The ephemeris used in computing the phases was that of Pigulski \& Jerzykiewicz (1988), i.e.

Min. light $=$ HJD $2439054.568+12.09684 E$.

In Fig. 6, the $y$ residuals are shown in a limited range of orbital phase around the primary eclipse (lower panel) and those around the phase of the secondary mid-eclipse, predicted by the spectroscopic elements from solution IV of L01 (upper panel). No secondary eclipse

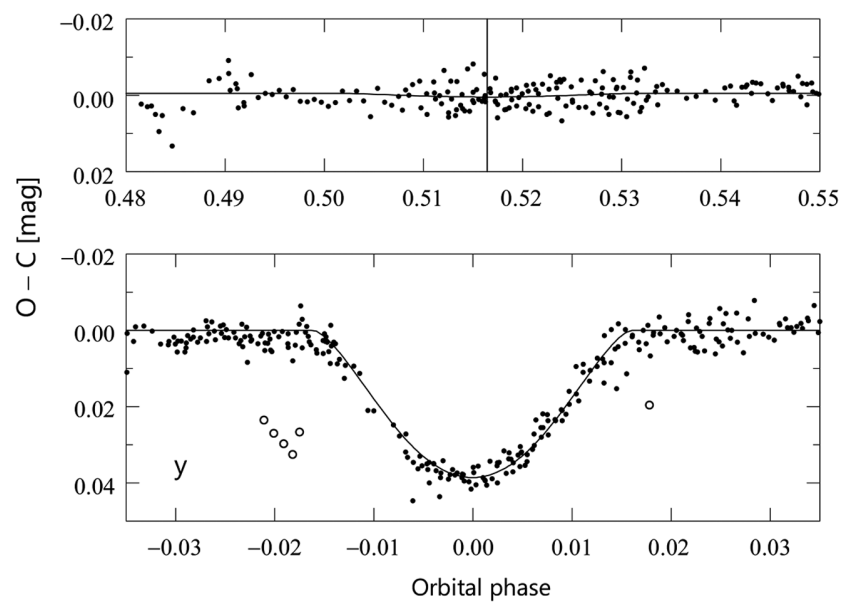

Figure 6. The $y$ residuals from the five-term pulsational solution in a limited interval of orbital phase around the primary and secondary eclipse. The vertical line in the upper panel indicates the predicted epoch of the secondary mid-eclipse. The other lines are fragments of the synthetic light curve, computed as described in the text. The open circles are deviant points not included in the analysis.

can be detected: the mean $y$ residual in the \pm 0.008 phase interval around the predicted mid-eclipse epoch amounts to $0.2 \pm 0.4 \mathrm{mmag}$. Clearly, the secondary component is much fainter than the primary.

In an attempt to derive the parameters of $16 \mathrm{Lac}$, we used Etzel's (1981) computer program EBOP. The program, based on the Nelson-Davis-Etzel model (Nelson \& Davis 1972; Popper \& Etzel 1981), is well suited for dealing with detached systems such as the present one. The spectroscopic parameters $\omega$ and $e$, needed to run the program, were taken from solution IV of L01. The components were assumed to be spherical because the secondary component's mass is much smaller than that of the primary and the system is well detached: for any reasonable assumption about the primary component's mass, the mass ratio would be equal to about 0.13 and the semimajor axis of the very nearly circular relative orbit, to about $50 \mathrm{R}_{\odot}$ or eight primary component's radii. However, we included reflected light from the secondary because a trace of a reflection effect can be seen in Fig. 5, especially in $y$ and $v$, in agreement with the results of the frequency analysis (see the fourth and the penultimate paragraphs of Section 3). The primary's limb-darkening coefficients were interpolated from table 2 of Walter Van Hamme ${ }^{1}$ for $T_{\text {eff }}=22500 \mathrm{~K}, \log g=3.85$ (see Section 5.1) and $[\mathrm{M} / \mathrm{H}]=0$ (Thoul et al. 2003; Niemczura \& Daszyńska-Daszkiewicz 2005). Unfortunately, since nothing is known about the secondary component except that it is much fainter than the primary, the central surface brightness of the secondary, $J_{\mathrm{s}}$, which EBOP uses as a fundamental parameter, must be derived indirectly. In units of the central surface brightness of the primary, we have

$J_{\mathrm{s}}=k^{-2} \frac{\left(1-u_{\mathrm{p}} / 3\right) l_{\mathrm{s}}}{\left(1-u_{\mathrm{s}} / 3\right) l_{\mathrm{p}}}$

where $l_{\mathrm{p}}$ and $l_{\mathrm{s}}$ are the normalized lights of the primary and the secondary, respectively, $u_{\mathrm{p}}$ and $u_{\mathrm{s}}$ are the limb darkening coefficients, and $k=r_{\mathrm{s}} / r_{\mathrm{p}}$ is the ratio of the radii. For $y$, we have

$\log \frac{l_{\mathrm{s}}}{l_{\mathrm{p}}}=2 \log k+4 \log k_{\mathrm{T}}+\Delta B C / 2.5$,

${ }^{1}$ http://www2.fiu.edu/ vanhamme/limdark.htm, see also Van Hamme (1993) 
where $k_{\mathrm{T}}=T_{\text {eff,s }} / T_{\text {eff,p }}$ is the ratio of the effective temperatures of the components and $\triangle B C=B C_{\mathrm{s}}-B C_{\mathrm{p}}$ is the difference of the bolometric corrections. Introducing $l_{\mathrm{s}} / l_{\mathrm{p}}$ from this equation into equation (3) we get

$J_{\mathrm{s}}=\frac{1-u_{\mathrm{p}} / 3}{1-u_{\mathrm{s}} / 3} k_{\mathrm{T}}^{4} 10^{\Delta B C / 2.5}$.

The bolometric correction of the primary component was taken from table 3 of Flower (1996) for $T_{\text {eff,p }}=22500 \mathrm{~K}$. Assuming $T_{\text {eff,s, }}$, we read the secondary's bolometric correction from the same table as above. Assuming further $\log g=4.0$ and $[\mathrm{M} / \mathrm{H}]=0$, we read the secondary's limb-darkening coefficients from table 2 of Walter Van Hamme. Setting the integration ring size to $1^{\circ}$ and the remaining parameters to their default EBOP values, we run the program for several values of $T_{\text {eff,s }}$ with $r_{\mathrm{p}}$, the relative radius of the primary component, $i$, the inclination of the orbit, $k$, the ratio of the radii, and $S_{\mathrm{s}}$, the reflected light from the secondary as unknowns. As data, we used the $y$ residuals (see Fig. 5, bottom panel). Unfortunately, we failed to find a solution which would converge. Convergent solutions were obtained if one of the first three unknowns, $r_{\mathrm{p}}, i$ or $k$, was fixed. After a number of trials, we decided to fix $k$, leaving $r_{\mathrm{p}}, i$, and $S_{\mathrm{s}}$ as the unknowns. For a given $k$, identical triples of $r_{\mathrm{p}}, i$, and $S_{\mathrm{s}}$ were obtained for different $T_{\text {eff,s }}$, i.e. different $J_{\mathrm{s}}$. For example, for $k=0.23, r_{\mathrm{p}}$ was equal to $0.1278 \pm 0.0015$ and $i$ was $82.93 \pm 0.10$, the same for $T_{\text {eff,s }}=5000$ and $6050 \mathrm{~K} ; S_{\mathrm{s}}$ was equal to $0.00023 \pm 0.00005$ for $5000 \mathrm{~K}$, and to $0.00025 \pm 0.00005$ for $6050 \mathrm{~K}$. The synthetic light curves computed from these solutions were very nearly identical everywhere but around the secondary eclipse: the computed depth of the secondary eclipse was equal to $0.1 \mathrm{mmag}$ for $T_{\text {eff, }}=5000 \mathrm{~K}$, while it was $0.7 \mathrm{mmag}$ for $T_{\mathrm{eff}, \mathrm{s}}=6050 \mathrm{~K}$. The mean residual in the \pm 0.008 phase interval around the predicted mid-eclipse epoch amounted to $0.3 \pm 0.4$ and $-0.2 \pm 0.4 \mathrm{mmag}$ for 5000 and $6050 \mathrm{~K}$, respectively. The mean residual was equal to $0.0 \pm 0.4 \mathrm{mmag}$ for $5750 \mathrm{~K}$, and the computed depth of the secondary eclipse was then $0.5 \mathrm{mmag}$. For this computed depth of the secondary eclipse, the mean residual was equal to $0.0 \pm 0.4$ mmag regardless of $k$.

\subsection{Evolutionary state of the secondary component}

For a range of $k$, synthetic light curves computed from the solutions with an assumed depth of the secondary eclipse are indistinguishable from one another. Each solution yields an abscissa for plotting the secondary component in the HR diagram. For an assumed mass of the primary component, one can also have the secondary's radius in absolute units from $r_{\mathrm{p}}, i$ and the spectroscopic elements $K_{1}$ and $e$, and therefore, the secondary's ordinate in the HR diagram. For three values of the assumed depth of the secondary eclipse, $0.1,0.5$ and $0.7 \mathrm{mmag}$, and the primary component's mass $M_{\mathrm{p}}=10 \mathrm{M}_{\odot}$, positions of the secondary component in the HR diagram are shown in Fig. 7 for a range of $k$. For the range of $k$ shown in Fig. 7, the assumption of $M_{\mathrm{p}}=10 \mathrm{M}_{\odot}$ implies $1.30 \leq M_{\mathrm{s}}<1.31 \mathrm{M}_{\odot}$. Therefore, without making noticeable errors, we can compare the HRdiagram positions of the secondary component with $M=1.30 \mathrm{M}_{\odot}$ evolutionary tracks. The evolutionary tracks plotted in the figure are the $1.30 \mathrm{M}_{\odot}, Y=0.265, Z=0.0175$ Pisa pre-main-sequence (pre-MS) tracks (Tognelli, Prada Moroni \& Degl'Innocenti 2011, http://astro.df.unipi.it/stellar-models/). The tracks were computed using the mixing-length theory of convection with three values of the mixing length $l=\alpha H_{\mathrm{p}}$, where $\alpha$ is the mixing-length parameter and $H_{\mathrm{p}}$ is the pressure scaleheight. The mid-value of $l(\alpha=1.68)$ was calibrated by means of the Pisa standard solar model (for further details see Tognelli et al. 2011). The thickened segment of the

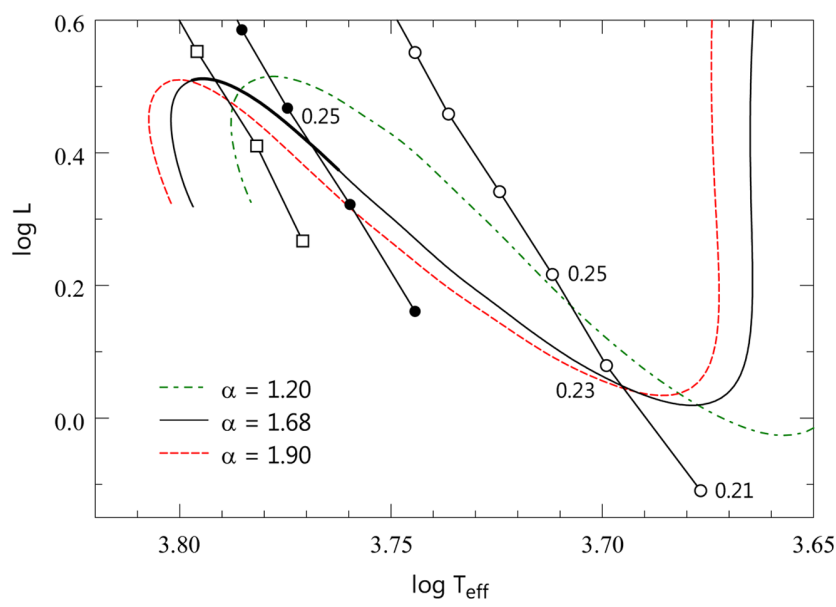

Figure 7. The HR-diagram positions of the secondary component of $16 \mathrm{Lac}$ for three series of eclipse solutions, obtained with the ratio of the radii $k=r_{\mathrm{s}} / r_{\mathrm{p}}$ as the parameter and assuming the depth of the secondary eclipse to be equal to $0.1 \mathrm{mmag}$ (open circles), $0.5 \mathrm{mmag}$ (dots) and 0.7 mmag (open squares). The open circles correspond to $k$ from 0.21 to 0.31 with a step of 0.02 , the dots, to $k$ from 0.21 to 0.27 with a step of 0.02 , and the open squares, to $k$ equal to $0.21,0.23$, and 0.25 ; three open circles and one dot are labelled with their values of $k$. Also shown are Pisa $1.3 \mathrm{M}_{\odot}$ pre-MS evolutionary tracks for three values of the mixing-length parameter $\alpha$ (the solid line, the green dash-dotted line and the red dashed line). The thickened segment of the $\alpha=1.68$ track indicates the age of $16.3 \pm 1.3 \mathrm{Myr}$, i.e. the evolutionary age of the primary component (see Section 5.2).

$\alpha=1.68$ track indicates the age within $1 \sigma$ of $16.3 \mathrm{Myr}$, the evolutionary age of the primary component (see Section 5.2). Since the duration of the pre-MS phase of the primary component's evolution is of the order of $0.1 \mathrm{Myr}$, we conclude that the secondary component is in the pre-MS contraction phase, the same conclusion as that reached long ago by Pigulski \& Jerzykiewicz (1988). Now, the points of intersection of the three series of the eclipse solutions in Fig. 7 with the pre-MS tracks constrain the ratio of the radii to $0.23 \lesssim k \lesssim 0.27$. If the mass of the primary component were assumed to be equal to $M_{\mathrm{p}}=8.8 \mathrm{M}_{\odot}$, corresponding to $M_{\mathrm{s}} \approx 1.20 \mathrm{M}_{\odot}$, the constraints would be $0.21 \lesssim k \lesssim 0.25$. If $M_{\mathrm{p}}=11.2 \mathrm{M}_{\odot}\left(M_{\mathrm{s}}\right.$ $\left.\approx 1.40 \mathrm{M}_{\odot}\right), 0.23 \lesssim k \lesssim 0.29$. Thus, for $8.8 \leq M_{\mathrm{p}} \leq 11.2 \mathrm{M}_{\odot}$ we get $0.21 \lesssim k \lesssim 0.29$. Over this range of $k$, the relative radius of the primary component, $r_{\mathrm{p}}$, is a monotonically increasing function of $k$, while the inclination of the orbit, $i$, is monotonically decreasing with $k$, and both are virtually independent of the assumed depth of the secondary eclipse. Thus, from the last inequality we have $0.125 \lesssim r_{\mathrm{p}} \lesssim 0.132$ and $83^{\circ} .4 \gtrsim i \gtrsim 82^{\circ}$. 0 . More importantly, we can also obtain the lower and upper bound of the logarithmic surface gravity of the primary component: $3.78 \lesssim \log g_{\mathrm{p}} \lesssim 3.87$. The formal standard deviations of $r_{\mathrm{p}}, i$ and $\log g_{\mathrm{p}}$, equal to $0.0013,0.10$ and 0.011 dex, respectively, are - not surprisingly - much smaller than the allowed ranges of $r_{\mathrm{p}}, i$ and $\log g_{\mathrm{p}}$.

The eclipse solutions which predict the depth of the secondary eclipse to be equal to $0.5 \mathrm{mmag}$ yield synthetic light curves which best fit the data around the secondary eclipse (see the end of the last paragraph of Section 4.1) while they fit the data elsewhere as well as do the other solutions. The fact that the corresponding line in Fig. 7 (the solid line with dots) crosses the $\alpha=1.68$ evolutionary track at an evolutionary age within the range of the evolutionary age of the primary component (see Section 5.2) is encouraging. It would be worthwhile to carry out space photometry of $16 \mathrm{Lac}$ in order to find out whether the depth of the secondary eclipse is indeed close 
to $0.5 \mathrm{mmag}$. In any case, space photometry will be necessary to better constrain the range of $k$, and therefore the ranges of $r_{\mathrm{p}}, i$ and $\log g_{\mathrm{p}}$.

\section{FUNDAMENTAL PARAMETERS}

\subsection{The effective temperature and the surface gravity}

The effective temperature and surface gravity of 16 Lac can be obtained from the Strömgren indices using several photometric calibrations available in the literature. The $c_{1}$ index from Hauck \& Mermilliod (1998), corrected for the interstellar reddening in the standard way (Crawford 1978), yielded the following values of $T_{\text {eff }}$ (with the calibration referenced in the parentheses after each value): 22435 K (Davis \& Shobbrook 1977), 22580 K (UVBYBETA ${ }^{2}$ ), $22430 \mathrm{~K}$ (Sterken \& Jerzykiewicz 1993), and $22635 \mathrm{~K}$ (Balona 1994). In the case of the Balona (1994) calibration, the $\beta$ index was also needed in addition to $c_{0}$. Taking a straight mean of these values, we get $T_{\text {eff }}=22520 \mathrm{~K}$, with a formal standard error equal to $50 \mathrm{~K}$. The latter number is so small because the four photometric calibrations are not independent; they all rely heavily on the OAO-2 absolute flux calibration of Code et al. (1976). Realistic standard deviations of the effective temperatures of early-type stars, estimated from the uncertainty of the absolute flux calibration, amount to about 3 per cent (Napiwotzki et al. 1993; Jerzykiewicz 1994) or $680 \mathrm{~K}$ for the $T_{\text {eff }}$ in question. Thus, $T_{\text {eff }}$ of $16 \mathrm{Lac}$, obtained from the Strömgren indices, is equal to $22520 \pm 680 \mathrm{~K}$. The most recent spectroscopic determinations of $T_{\text {eff }}$ include $22900 \pm 1000 \mathrm{~K}$ (Thoul et al. 2003), $21500 \pm 750 \mathrm{~K}$ (Prugniel, Vauglin \& Koleva 2011) and $23000 \pm 200 \mathrm{~K}$ (Nieva \& Przybilla 2012). A straight mean of these numbers is equal to $22470 \pm 480 \mathrm{~K}$, in surprisingly good agreement with the photometric value. We shall adopt $22500 \pm 600 \mathrm{~K}$ as the $T_{\text {eff }}$ of $16 \mathrm{Lac}$.

The logarithmic surface gravity of 16 Lac derived from $\beta$ and $c_{0}$ turned out to be equal to 3.93 (UVBYBETA) and 3.90 (Balona 1994). The good agreement between these values may be misleading: according to Napiwotzki et al. (1993), the uncertainty of photometric surface gravities of hot stars is equal to $0.25 \mathrm{dex}$. We conclude that the photometric $\log g$ of $16 \mathrm{Lac}$ is equal to $3.90 \pm 0.25$. The spectroscopic values of $\log g$ are equal to $3.80 \pm 0.20$ (Thoul et al. 2003), $3.75 \pm 0.17$ (Prugniel et al. 2011) and $3.95 \pm 0.05$ (Nieva \& Przybilla 2012). A straight mean of these numbers is equal to 3.83 , with a standard error equal to $0.06 \mathrm{dex}$. We shall adopt $3.85 \pm 0.15$ as the $\log g$ of $16 \mathrm{Lac}$, where the adopted standard deviation, equal to the median of the standard deviations of the individual $\log g$ values, is a compromise between the standard deviation of the photometric $\log g$ and that of the spectroscopic $\log g$ of Nieva \& Przybilla (2012). Note that because of negligible brightness of the secondary component, the $T_{\text {eff }}$ and $\log g$ we adopted pertain to the primary component.

\subsection{The effective temperature - surface gravity diagram}

In Fig. 8, the primary component of $16 \mathrm{Lac}$ is plotted in the $\log T_{\text {eff }}-$ $\log g$ plane using the effective temperature and surface gravity from

\footnotetext{
${ }^{2}$ A FORTRAN program based on the grid published by Moon \& Dworetsky (1985). Written in 1985 by T.T. Moon of the University London and modified in 1992 and 1997 by R. Napiwotzki of Universitaet Kiel (see Napiwotzki, Schönberner \& Wenske 1993).
}

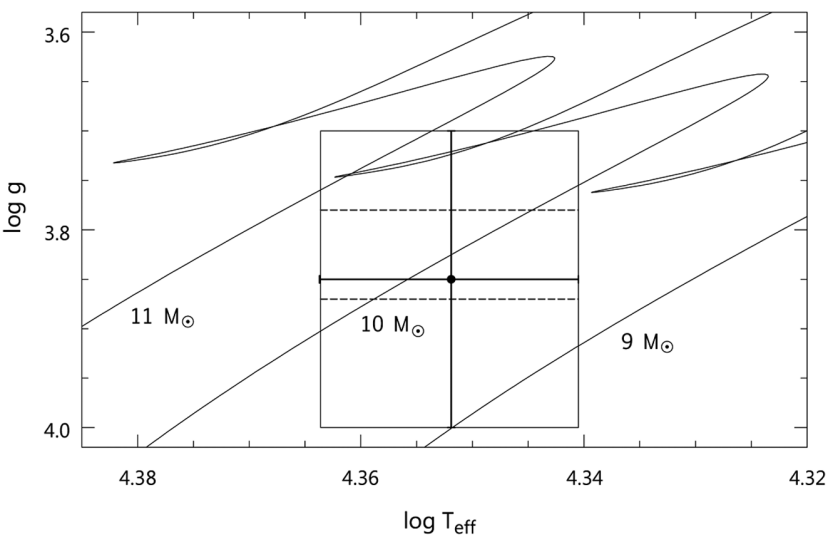

Figure 8. The primary component of $16 \mathrm{Lac}$ plotted in the effective temperature - surface gravity plane using $T_{\text {eff }}$ and $\log g$ from Section 5.1 (dot with error bars). The dashed horizontal lines indicate the lower and upper bound of $\log g_{\mathrm{p}}$, obtained in Section 4.2 from the eclipse solutions and pre-MS evolutionary tracks. The 9,10 and $11 \mathrm{M}_{\odot}$ evolutionary tracks are explained in the text.

Section 5.1 (dot with error bars). The dashed horizontal lines indicate the lower and upper bound of $\log g_{\mathrm{p}}$, obtained in Section 4.2 from the eclipse solutions. Also shown are evolutionary tracks computed by means of the Warsaw-New Jersey evolutionary code (see e.g. Pamyatnykh et al. 1998), assuming the initial abundance of hydrogen $X=0.7$ and the metallicity $Z=0.015$, the OPAL equation of state (Rogers \& Nayfonov 2002) and the OP opacities (Seaton 2005) for the latest heavy element mixture of Asplund et al. (2009). For lower temperatures, the opacity data were supplemented with the Ferguson tables (Ferguson, Alexander \& Allard 2005; Serenelli et al. 2009). We assumed no convective-core overshooting and $V_{\text {rot }}=20 \mathrm{~km} \mathrm{~s}^{-1}$ on the zero-age main sequence, a value consistent with the observed $V_{\text {rot }} \sin i$ (Głęocki, Gnaciński \& Stawikowski 2000) under the assumption that the rotation and orbital axes are aligned. The effect of rotation on $\log g$ was taken into account by subtracting the centrifugal acceleration, amounting in the present case to about 0.001 dex.

The evolutionary mass at the position of the dot in Fig. 8, $M_{\mathrm{ev}}=9.8 \pm 1.3 \mathrm{M}_{\odot}$; the standard deviation of $\log g$ is responsible for the standard deviation of $M_{\mathrm{ev}}$. If the lower and upper bound of $\log g_{\mathrm{p}}$ were used, the result would be $9.3 \lesssim M_{\mathrm{ev}} \lesssim 10.8 \mathrm{M}_{\odot}$. According to Nieva \& Przybilla (2012), $M_{\mathrm{ev}}=9.8 \pm 0.3 \mathrm{M}_{\odot}$. In this case, the small standard deviation of $M_{\mathrm{ev}}$ is the consequence of the small standard deviations these authors assign to their spectroscopic $T_{\text {eff }}$ and $\log g$. The evolutionary age of 16 Lac, obtained from Fig. 8, amounts to $16.3 \pm 1.5 \mathrm{Myr}$, in surprisingly good agreement with the Blaauw's (1964) estimate of the age of Lac OB1a. The most recent asteroseismic analysis of the star (Thoul et al. 2003) has led to a mass of $9.62 \pm 0.11 \mathrm{M}_{\odot}$ and an age of $15.7 \mathrm{Myr}$. Clearly, the accuracy of the asteroseismic values is much higher than that attainable by either the photometric or spectroscopic method. However, the sensitivity of the asteroseismic values to the details of modelling needs to be examined. We leave this for a future paper.

\section{THE HARMONIC DEGREE OF THE THREE HIGHEST AMPLITUDE MODES}

\subsection{From the uvy data}

Our data are the most extensive photometric observations of $16 \mathrm{Lac}$ ever obtained. In addition, the three photometric passbands we used 
Table 2. The $u y$ and $u v$ amplitude ratios for the three highest amplitude modes. In the case of $f_{2}$, the HJD 245855.4 values were omitted in computing the weighted means.

\begin{tabular}{|c|c|c|c|c|c|c|}
\hline \multirow[b]{2}{*}{ HJD-245 2800} & \multicolumn{2}{|c|}{$f_{1}$} & \multicolumn{2}{|c|}{$f_{2}$} & \multicolumn{2}{|c|}{$f_{3}$} \\
\hline & $A_{y} / A_{u}$ & $A_{v} / A_{u}$ & $A_{y} / A_{u}$ & $A_{v} / A_{u}$ & $A_{y} / A_{u}$ & $A_{v} / A_{u}$ \\
\hline 55.4 & $0.565 \pm 0.078$ & $0.560 \pm 0.082$ & $0.324 \pm 0.870$ & $0.270 \pm 0.900$ & $0.643 \pm 0.088$ & $0.697 \pm 0.096$ \\
\hline 107.1 & $0.494 \pm 0.040$ & $0.540 \pm 0.040$ & $0.697 \pm 0.108$ & $0.843 \pm 0.114$ & $0.649 \pm 0.040$ & $0.723 \pm 0.040$ \\
\hline 124.8 & $0.489 \pm 0.028$ & $0.567 \pm 0.030$ & $0.753 \pm 0.066$ & $0.816 \pm 0.072$ & $0.655 \pm 0.026$ & $0.719 \pm 0.028$ \\
\hline 135.1 & $0.505 \pm 0.034$ & $0.588 \pm 0.040$ & $0.743 \pm 0.072$ & $0.831 \pm 0.084$ & $0.647 \pm 0.028$ & $0.706 \pm 0.034$ \\
\hline 160.0 & $0.444 \pm 0.050$ & $0.494 \pm 0.056$ & $0.663 \pm 0.084$ & $0.676 \pm 0.088$ & $0.643 \pm 0.042$ & $0.685 \pm 0.044$ \\
\hline 198.4 & $0.440 \pm 0.060$ & $0.517 \pm 0.070$ & $0.758 \pm 0.056$ & $0.818 \pm 0.060$ & $0.676 \pm 0.046$ & $0.723 \pm 0.052$ \\
\hline Wt. Mean = & $0.488 \pm 0.021$ & $0.554 \pm 0.023$ & $0.734 \pm 0.035$ & $0.800 \pm 0.038$ & $0.652 \pm 0.020$ & $0.712 \pm 0.022$ \\
\hline
\end{tabular}

include one on the short-wavelength side of the Balmer jump and two in the Paschen continuum. Thus, we can derive the amplitude ratios, $A_{y} / A_{u}$ and $A_{v} / A_{u}$, and the phase differences, $\Phi_{y}-\Phi_{u}$ and $\Phi_{v}-\Phi_{u}$, which will be more accurate than any available before and sensitive to the harmonic degree of the pulsation modes. However, before computing the amplitudes and the phases we had to tackle the problem of the differences in the number and the time distribution between the $y, v$ and $u$ data. Since the $u$ data are fewer in number and less evenly distributed in time than the $y$ data, there were fewer $u$ segments than the $y$ segments (compare Fig. 3 with Fig. 2). Consequently, the mean epochs of the $u$ segments did not match those of the $y$ segments. To a smaller degree, this was also the case with the $v$ data. Because the amplitudes vary from one segment to another, the amplitude ratios computed using amplitudes from unmatched segments would be biased; the same goes for the phases and the phase differences. We therefore divided the $y$ and $v$ data into new segments, in most cases different from those we formed in Section 3. In the new segments, the initial and final epochs and the time distribution of the data matched those of the $u$ segments as closely as the data allowed. Then, the amplitudes and the phases in each segment were derived by fitting equation (1) with $N=5$ to the data; the frequencies $f_{i}(i=1, \ldots, 4)$ and $f_{5}=f_{2}+f_{3}$ were the same as in Section 3. Finally, the amplitudes of the three highest amplitude modes from the matching segments were used to compute the amplitude ratios, and the phases, to compute the phase differences. In spite of the high quality of our photometry, standard deviations of the phase differences were rather large, rendering them useless for mode identification. The amplitude ratios and their weighted means are listed in Table 2. There is no evidence in the table for a time-variability of the amplitude ratios, a result consistent with the fact that we are dealing with normal pulsation modes. In computing the weighted means, we assumed weights inversely proportional to the squares of the standard deviations of the components. Standard deviations of the weighted means were computed by adding the standard deviations of the components in quadrature and dividing the sum by the number of components. Note that for $f_{2}$, the JD 2452855.4 values are deviant. This is because at this epoch the $f_{2}$ amplitudes were close to zero. In computing the weighted means, we omitted the JD 2452855.4 values.

A comparison of the observed amplitude ratios with the theoretical ones is presented in Fig. 9. The theoretical amplitudes were computed according to the zero-rotation formulae of DaszyńskaDaszkiewicz et al. (2002) using the non-adiabatic pulsational code of Dziembowski (1977) and Kurucz line-blanketed LTE model atmospheres (Kurucz 2004) for $[\mathrm{M} / \mathrm{H}]=0.0$ and the microturbulent velocity $\xi=2 \mathrm{~km} \mathrm{~s}^{-1}$. The remaining input parameters were the same as those used in computing the evolutionary tracks in Section 5.2. The calculations were carried out for $\log T_{\text {eff }}$ and $\log g$

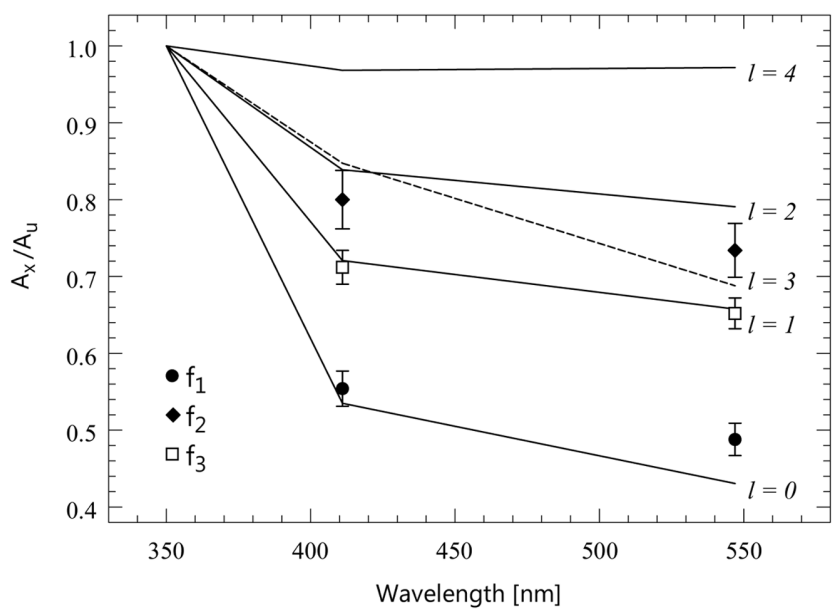

Figure 9. A comparison of the observed (symbols with error bars) and theoretical (lines) uvy amplitude ratios for the three highest amplitude pulsation modes of $16 \mathrm{Lac}, f_{1}, f_{2}$ and $f_{3}$. The observed amplitude ratios are the weighted means listed in the bottom line of Table 2. The theoretical amplitude ratios correspond to the dot in Fig. 8. The theoretical $\ell=3$ amplitude ratios are shown with the dashed line.

used to plot the dot in Fig. 8. The $\log g_{\mathrm{p}}$ obtained in Section 4.2, although more precise than $\log g$, was not used because it may be less accurate on account of being model-dependent. As can be seen from Fig. 9, $f_{1}$ should be identified with a radial mode, notwithstanding that the agreement between the observed and theoretical $A_{y} / A_{u}$ is problematic. The remaining two modes are non-radial with $\ell \leq 3$ because neither the $\ell=0$, nor the $\ell=4$ line fits their amplitude ratios. In the case of $f_{3}$, the observed and theoretical amplitude ratios agree to within $1 \sigma$ for $\ell=1$, while in the case of $f_{2}$, the observed $A_{v} / A_{u}$ falls about $1 \sigma$ below the $\ell=2$ and 3 lines while the observed $A_{y} / A_{u}$ lies half-way between the $\ell=2$ and 3 lines.

\subsection{From the Geneva $U B G$ data}

The data obtained with the Geneva filters (see Section 2) cover three intervals: JD 2452861.6 to JD 245 2872.7, JD 2452921.5 to JD 245 2949.5, and JD 2452971.3 to JD 245 2991.5. From the data in the latter two intervals we derived the amplitude ratios, $A_{G} / A_{U}$ and $A_{B} / A_{U}$ in the same way as from the $u v y$ data in Section 6.1. The amplitude ratios are listed in Table 3 . The weighted mean amplitude ratios are plotted as a function of the passbands' central frequency in Fig. 10. Also plotted are the theoretical amplitude ratios. The harmonic-degree identification of $\ell=0$ for $f_{1}$ and $\ell=1$ for $f_{3}$ inferred in Section 6.1 from the $u v y$ data is confirmed. In the case of $f_{2}, \ell=2$ fits now better than $\ell=3$. In addition, $\ell=3$ would be 
Table 3. The Geneva $U G$ and $U B$ amplitude ratios for the three highest amplitude modes.

\begin{tabular}{lcccccr}
\hline HJD-245 2800 & \multicolumn{2}{c}{$f_{1}$} & \multicolumn{2}{c}{$f_{2}$} & & $f_{3}$ \\
& $A_{G} / A_{U}$ & $A_{B} / A_{U}$ & $A_{G} / A_{U}$ & $A_{B} / A_{U}$ & $A_{G} / A_{U}$ & $A_{B} / A_{U}$ \\
\hline 135.7 & $0.477 \pm 0.086$ & $0.580 \pm 0.086$ & $0.727 \pm 0.155$ & $0.821 \pm 0.158$ & $0.647 \pm 0.060$ & $0.721 \pm 0.060$ \\
181.9 & $0.476 \pm 0.092$ & $0.563 \pm 0.090$ & $0.778 \pm 0.112$ & $0.833 \pm 0.108$ & $0.676 \pm 0.063$ & $0.717 \pm 0.060$ \\
Wt. Mean $=$ & $0.476 \pm 0.063$ & $0.572 \pm 0.062$ & $0.761 \pm 0.096$ & $0.829 \pm 0.096$ & $0.661 \pm 0.044$ & $0.719 \pm 0.043$ \\
\hline
\end{tabular}

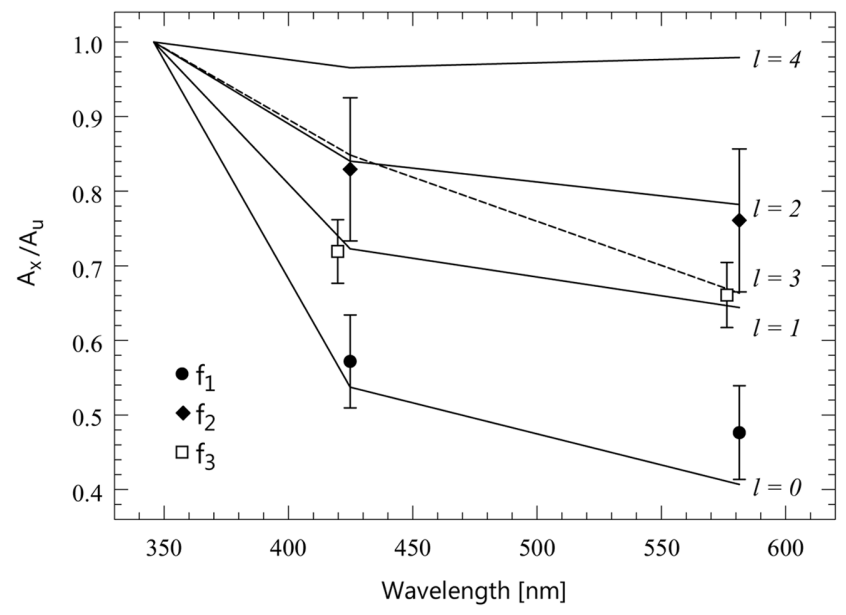

Figure 10. A comparison of the observed (symbols with error bars) and theoretical (lines) $U B G$ amplitude ratios for the three highest amplitude pulsation modes of $16 \mathrm{Lac}, f_{1}, f_{2}$ and $f_{3}$; the open square was shifted slightly along the abscissa to avoid overlap. The observed amplitude ratios are the weighted means listed in the bottom line of Table 3 . The theoretical amplitude ratios correspond to the dot in Fig. 8.

less satisfactory than $\ell=2$ because of the effect of cancellation in integrating over the stellar disc. However, the standard deviations of the $f_{2}$ amplitude ratios are rather large.

These harmonic-degree identifications, i.e. $\ell=0$ for $f_{1}, \ell=2$ or, less satisfactorily, $\ell=3$ for $f_{2}$, and $\ell=1$ for $f_{3}$, agree with the earlier identifications, based on the $U B V$ amplitude ratios and the $V$-amplitude to the RV-amplitude ratio (see Dziembowski \& Jerzykiewicz 1996). They have points in common with the spectroscopic identifications of Aerts et al. (2003a,b). An analysis of the line profiles of the $\mathrm{He}$ I $\lambda 6678 \AA$ line led Aerts et al. (2003a) to the conclusion that $f_{1}$ should be identified with a radial mode, $f_{2}$, with an $\ell=2, m=0$ mode, and $f_{3}$, with an $\ell=1, m=0$ mode. Subsequently, Aerts et al. (2003b) modified the harmonic-degree identification for $f_{3}$ to $\ell<3$. For $f_{2}$, the identification of Aerts et al. (2003b) is thus more specific than ours, while the reverse is true for $f_{3}$.

\section{LONG-TERM VARIATION OF THE AMPLITUDE AND PHASE OF THE LARGE-AMPLITUDE TERMS}

\subsection{The $f_{1}$ term}

All out-of-eclipse blue-filter observations of 16 Lac obtained throughout 1992 span an interval of over $40 \mathrm{yr}$ and consist of 6334 data points (see JP99). By supplementing these data with our out-of-eclipse $y$ observations, we formed a data set of 9384 points, spanning an interval of $53.4 \mathrm{yr}$. We shall refer to this set as $B \& y$. Subtracting the contribution of the $f_{i}$ and $f_{j}$ terms $(i, j=1$, $2,3, i \neq j$ ) from $B \& y$ resulted in three sets which we shall refer

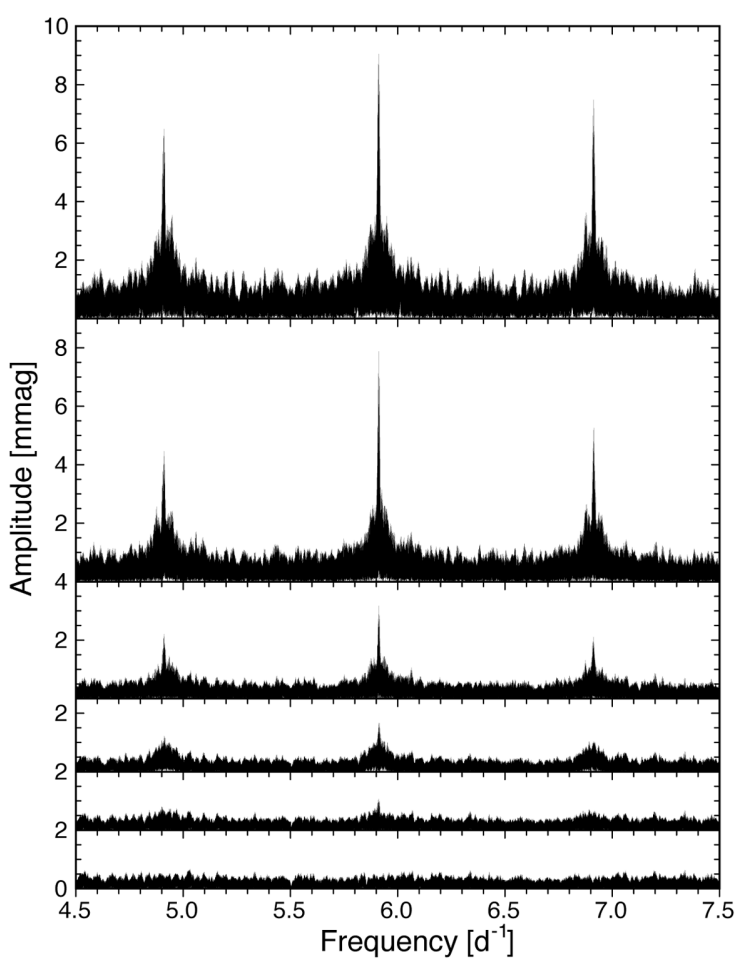

Figure 11. The amplitude spectra of $B \& y-23$, i.e. the 1950-1992 archival blue-filter data and our $y$-filter data combined, freed from the contribution of the $f_{2}$ and $f_{3}$ terms. Starting with the second panel from the top, the data were pre-whitened with (1) $f_{1,1}=5.911312 \mathrm{~d}^{-1}$, (2) $f_{1,1}$ and $f_{1,2}=$ $5.911275 \mathrm{~d}^{-1}$, (3) $f_{1,1}, f_{1,2}$ and $f_{1,3}=5.911350 \mathrm{~d}^{-1}$, (4) $f_{1,1}, f_{1,2}, f_{1,3}$ and $f_{1,4}=5.911230 \mathrm{~d}^{-1}$, and (5) $f_{1,1}, f_{1,2}, f_{1,3}, f_{1,4}$ and $f_{1,5}=5.911395 \mathrm{~d}^{-1}$.

to as $B \& y-23, B \& y-13$ and $B \& y-12$. We chose the archival blue-filter observations and the present $y$ observations because they are much more numerous than observations in other filters. In the following frequency analysis of the combined data, we shall neglect the difference between the blue and yellow pulsation amplitudes. This will lead to some amplitude smearing in the results reported in this and the two following sections. In 1965, when the amplitudes of the three high-amplitude terms were close to their maximum values, the difference between the $B$ and $V$ amplitudes amounted to $2.0 \pm 0.21,0.40 \pm 0.21$ and $0.70 \pm 0.20 \mathrm{mmag}$ for $f_{1}, f_{2}$ and $f_{3}$, respectively (see Jerzykiewicz 1993, tables 7 and 8). Thus, the amplitude smearing will be the largest (albeit far from severe) in the case of $f_{1}$ and very nearly negligible in the remaining cases.

The amplitude spectra of $B \& y-23$ are shown in Fig. 11. The abscissae of the highest peaks in the amplitude spectra are given in the caption to the figure and are listed in the second column of Table 4; the corresponding periods are given in column three. The amplitudes and phases with their formal standard deviations, obtained from a five-frequency least-squares fit of equation (1) to $B \& y-23$ are listed in columns four and five. The standard 
Table 4. Fine structure of the $f_{1}$ term.

\begin{tabular}{lccrc}
\hline$j$ & $f_{1, j}\left(\mathrm{~d}^{-1}\right)$ & $P_{1, j}(\mathrm{~d})$ & $A_{1, j}(\mathrm{mmag})$ & $\Phi_{1, j}(\mathrm{rad})$ \\
\hline 1 & 5.911312 & 0.1691672 & $12.45 \pm 0.09$ & $0.181 \pm 0.007$ \\
2 & 5.911275 & 0.1691682 & $9.06 \pm 0.09$ & $4.080 \pm 0.010$ \\
3 & 5.911350 & 0.1691661 & $3.27 \pm 0.09$ & $3.326 \pm 0.027$ \\
4 & 5.911230 & 0.1691695 & $2.26 \pm 0.09$ & $1.836 \pm 0.039$ \\
5 & 5.911395 & 0.1691648 & $1.35 \pm 0.09$ & $5.367 \pm 0.065$
\end{tabular}

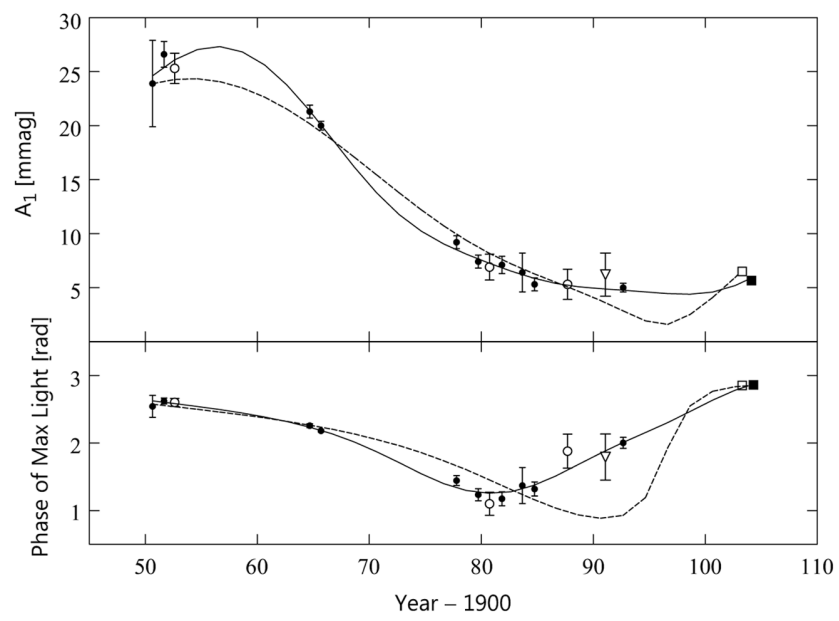

Figure 12. Upper panel: the amplitude of the $f_{1}$ term, $A_{1}$, computed from the parameters of the five-frequency fit of Table 4 (solid line), compared with the yearly mean blue-light 1950-1992 $A_{1}$ (the dots, circles and the triangle) and the mean $2003 y$ and $v A_{1}$ (the filled and empty square, respectively). The dots, circles and the triangle are from fig. 2 (top) of JP99 but with the error bars doubled. The 2003 error bars are not plotted because they would be of about the same size as the symbols. Also shown is the amplitude, computed using the parameters of a fit with the first three frequencies of Table 4 (dashed line). Lower panel: the same for the phase of maximum light of the $f_{1}$ term. In both panels, the 2003 symbols are shifted along the abscissa by \pm 0.5 yr to avoid overlap.

deviation of the fit amounted to $5.4 \mathrm{mmag}$. The epochs of observations were reckoned from HJD 2445784.

The five-frequency fit accounts very well for the long-term variation of the amplitude and phase of the $f_{1}$ term. This can be seen from Fig. 12 where the amplitude and the phase of maximum light computed from the parameters of Table 4 (solid lines) are compared with the yearly mean amplitudes and the yearly mean phases of maximum light (upper and lower panel, respectively). The observed and computed phases of maximum light, $\varphi_{\max }$, were obtained from the observed and computed epochs of maximum light, $\mathrm{HJD}_{\max }$, using the formula

$\varphi_{\max }=2 \pi\left[E-\left(\mathrm{HJD}_{\max }-\mathrm{HJD}_{0}\right) \mathrm{f}\right]$,

where $E$ is the number of cycles which elapsed from an arbitrary initial epoch $\operatorname{HJD}_{0}$ and $f=f_{1,1}$ from Table 4 . The computed amplitude and phase of maximum light agree also with the nightly amplitudes and the nightly phases of maximum light. This is illustrated in the upper half of Fig. 13 where the solid lines of Fig. 12 are plotted together with the $1965 B$-filter amplitudes from JP96 and the 1965 $B$-filter phases of maximum light. The agreement between the computed amplitude and the $y$ and $v$ amplitudes derived in Section 3 is also satisfactory (see the upper panel of the lower half of Fig. 13). The same goes for the computed and observed phases of maximum light (the lower panel of the lower half of the figure). Note that the lines in Figs 12 and 13 were not fitted to the points shown in the
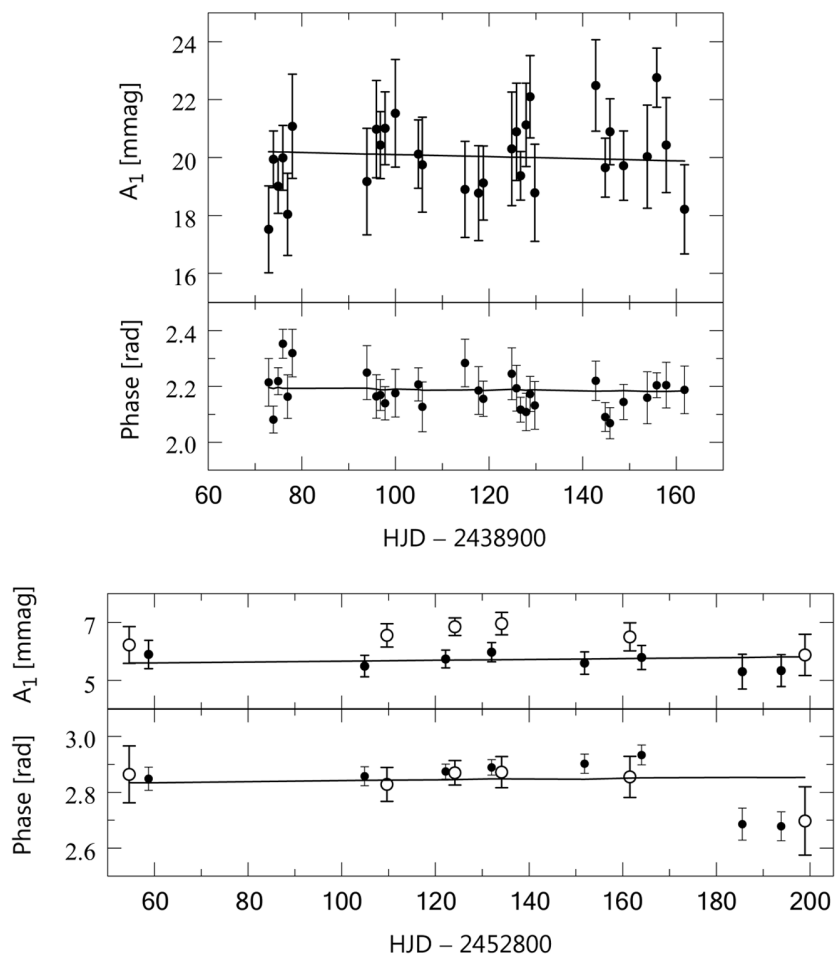

Figure 13. Upper half, upper panel: the amplitude of the $f_{1}$ term, $A_{1}$, computed from the parameters of the five-frequency fit of Table 4 (solid line), compared with the nightly $1965 B$-filter $A_{1}$ from JP96 (dots). Lower half, upper panel: the same for the $y$ and $v A_{1}$ derived in Section 3 (the dots and circles, respectively). Upper half, lower panel: the phase of maximum light of the $f_{1}$ term, computed from the parameters of the five-frequency fit of Table 4 (solid line), compared with the $1965 B$-filter phases of maximum light (dots). Lower half, lower panel: the same for the $y$ and $v$ phases of maximum light (the dots and circles, respectively).

figures but were computed independently from the parameters of the five-frequency fit. From Fig. 12, it is also clear that the three first frequencies of Table 4 alone would be insufficient to account for the variation of the phase, especially around 1900. This is an important conclusion because the first three periods in Table $4, P_{1,1}$, $P_{1,2}$ and $P_{1,3}$, are very nearly equal to the periods $P_{1}, P_{1}^{-}$and $P_{1}^{+}$ of L01, mentioned in the Introduction: the differences (in the sense 'Table 4 minus L01') amount to 0.000000 13, 0.00000011 and $0.00000005 \mathrm{~d}$, respectively.

The first three frequencies of Table 4 form a very nearly equally spaced triplet, $f_{1,2}, f_{1,1}, f_{1,3}$, with a mean spacing equal to $0.0137 \mathrm{yr}^{-1}$. The remaining frequencies, $f_{1,4}$ and $f_{1,5}$, flank the triplet at a distance of $0.0164 \mathrm{yr}^{-1}$ from the first and the last frequency of the triplet, respectively. The triplet's spacing implies a time-scale of $73 \mathrm{yr}$ for the long-term variation of the amplitude and phase of the $f_{1}$ term, while accounting for the non-sinusoidal shape of the variation seen in Fig. 12 requires all five components. Note that the reciprocal of the time-span of the data is equal to $1 / 53.4=0.0187 \mathrm{yr}^{-1}$, so that the spacings of the adjacent frequencies of the quintuplet amount to about $3 / 4$ of the formal frequency resolution of the data. That they could be resolved nevertheless is due to their unequal amplitudes.

\subsection{The $f_{2}$ term}

Frequency analysis of $B \& y-13$ yielded four frequencies before the noise prevented detecting further frequencies. However, a four-frequency fit did not account very well for the variation of the 
Table 5. Fine structure of the $f_{2}$ term.

\begin{tabular}{lcccrc}
\hline$j$ & $f_{2, j}\left(\mathrm{~d}^{-1}\right)$ & $P_{2, j}(\mathrm{~d})$ & $A_{2, j}(\mathrm{mmag})$ & \multicolumn{1}{c}{$B_{2, j}\left(\mathrm{mmag} \mathrm{d}^{-1}\right)$} & $\Phi_{2, j}(\mathrm{rad})$ \\
\hline 1 & 5.855574 & 0.1707775 & $6.828 \pm 0.093$ & $-0.000173 \pm 0.000021$ & $5.017 \pm 0.013$ \\
2 & 5.852967 & 0.1708535 & $3.592 \pm 0.096$ & $0.000047 \pm 0.000016$ & $5.199 \pm 0.028$ \\
3 & 5.852904 & 0.1708554 & $3.008 \pm 0.090$ & $-0.000074 \pm 0.000023$ & $5.483 \pm 0.028$ \\
4 & 5.858222 & 0.1707003 & $2.164 \pm 0.096$ & $0.000108 \pm 0.000016$ & $3.836 \pm 0.043$ \\
\hline
\end{tabular}

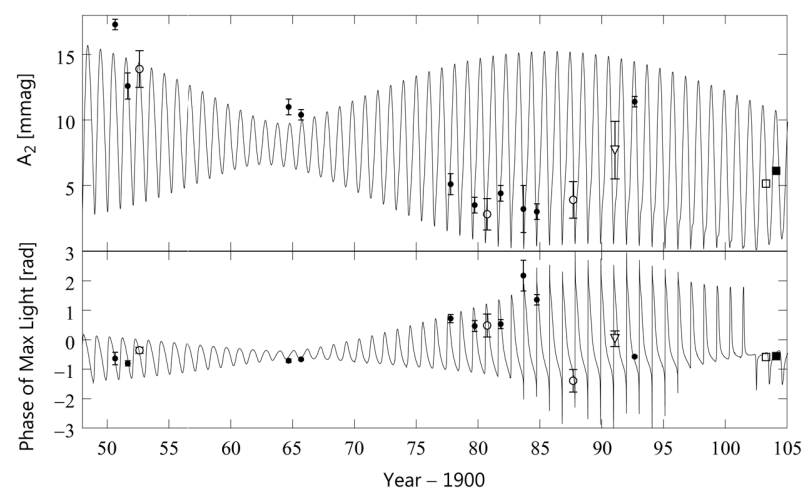

Figure 14. Upper panel: the amplitude of the $f_{2}$ term, $A_{2}$, computed from the parameters of the four-frequency fit of Table 5 (solid line), compared with the yearly mean blue-light $1950-1992 A_{2}$ (the dots, circles and the triangle) and the mean $2003 y$ and $v A_{2}$ (the filled and empty square, respectively). The dots, circles and the triangle are from fig. 3 (top) of JP99 but with the error bars doubled. The 2003 error bars are not plotted because they would be of about the same size as the symbols. Lower panel: the same for the phase of maximum light of the $f_{2}$ term. In both panels, the 2003 symbols were shifted along the abscissa by $\pm 0.5 \mathrm{yr}$ to avoid overlap.

amplitude and phase of the $f_{2}$ term. After a number of trials, we found that the fit improved when the amplitudes were assumed to vary uniformly with time, i.e. when constant amplitudes in the observational equations were replaced by $A_{2, j}+B_{2, j} t$. The parameters of a least-squares fit with the amplitudes modified in this way are listed in Table 5. The standard deviation of the fit amounted to $5.6 \mathrm{mmag}$. The epochs of observations were reckoned from HJD 2445784. A comparison of the amplitudes and phases of maximum light computed from the parameters of Table 5 (solid lines) with the observed amplitudes and phases of maximum light is shown in Figs 14 and 15. The observed and computed phases of maximum light were obtained from the observed and computed maxima using equation (5) with $f=f_{2,1}$ from Table 5. The agreement between the computed and observed amplitudes and phases of maximum light of the $f_{2}$ term seen in Figs 14 and 15 is less satisfactory than was the case for $f_{1}$ (Figs 12 and 13).

As can be seen from Table 5, $f_{2,1}-f_{2,2}=0.002607$ and $f_{2,4}-f_{2,1}=0.002648 \mathrm{~d}^{-1}$, so that $f_{2,2}, f_{2,1}$ and $f_{2,4}$ form a very nearly equidistant frequency triplet, with a mean separation equal to $0.002628 \mathrm{~d}^{-1}$. Although this number is close to $1 \mathrm{yr}^{-1}$, it is not an artefact because the aliases were removed in our procedure of pre-whitening. The beat period corresponding to the mean separation of the triplet is equal to $380.5 \mathrm{~d}$, a value about 10 per cent greater than the beat-period between the L01 periods $P_{2}$ and $P_{2}^{+}$. The difference between $f_{2,2}$ and $f_{2,3}$ is equal to $0.000063 \mathrm{~d}^{-1}$ or $0.023 \mathrm{yr}^{-1}$, implying a time-scale of $43 \mathrm{yr}$. The latter number is close to the time-scale of the variation of $A_{2}$ derived by JP99 from the 1950-1992 data.
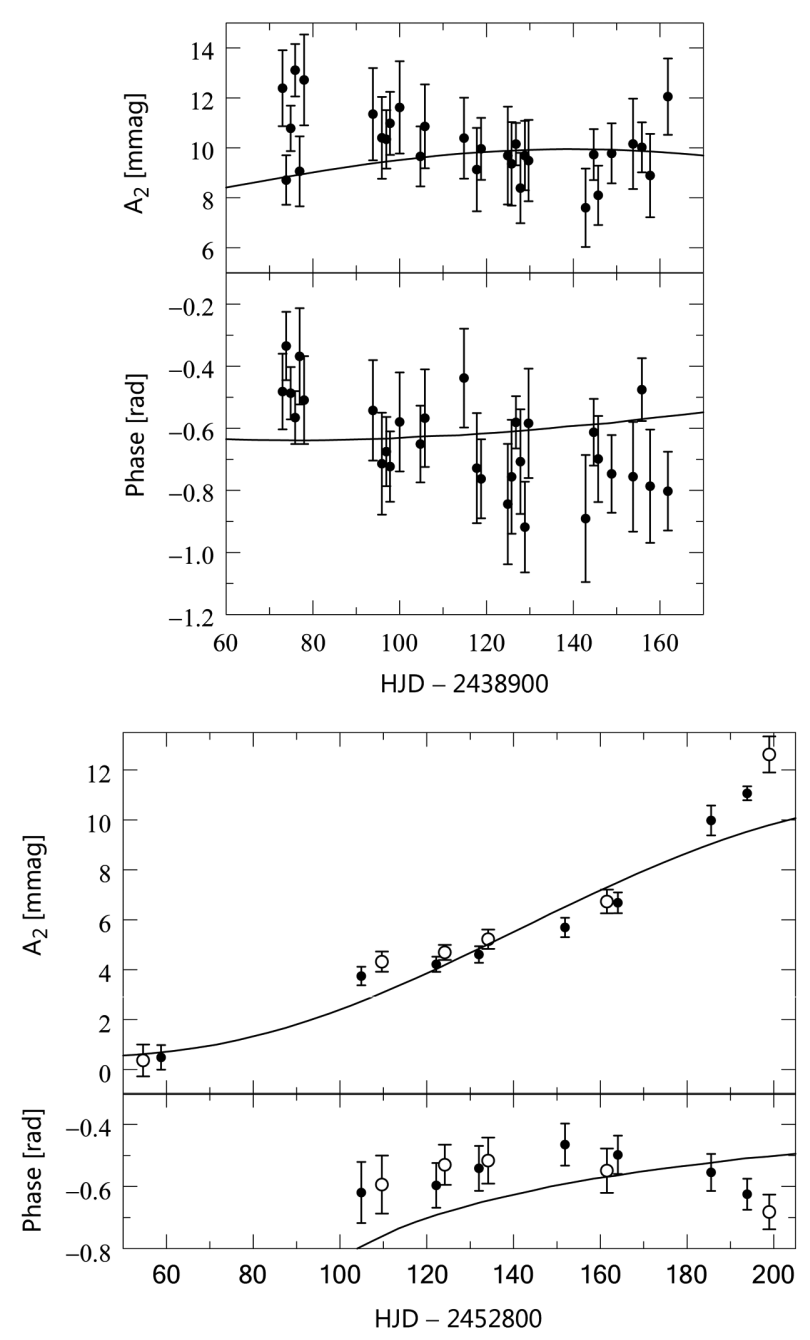

Figure 15. Upper half, upper panel: the amplitude of the $f_{2}$ term, $A_{2}$, computed from the parameters of the four-frequency fit of Table 5 (solid line), compared with the nightly $1965 B$-filter $A_{2}$ from JP96 (dots). Lower half, upper panel: the same for the $y$ and $v A_{2}$ derived in Section 3 (the dots and circles, respectively). Upper half, lower panel: the phase of maximum light of the $f_{2}$ term, computed from the parameters of the five-frequency fit of Table 5 (solid line), compared with the $1965 B$-filter phases of maximum light (dots). Lower half, lower panel: the same for the $y$ and $v$ phases of maximum light (the dots and circles, respectively). The first-segment $y$ and $v$ phases of maximum light, equal to $-1.6 \pm 1.0$ and $1.7 \pm 1.7 \mathrm{rad}$, respectively, are not shown.

\subsection{The $f_{3}$ term}

As we mentioned in the Introduction, the $f_{3}$ term was found by JP96 to be a doublet. Using all blue-filter observations of 16 Lac available at the time, JP99 determined the doublet frequencies to be $f_{3,1}=5.5025779 \pm 0.0000005$ and $f_{3,2}=5.5040531 \pm 0.0000008 \mathrm{~d}^{-1}$. Slightly different frequencies, 
Table 6. Fine structure of the $f_{3}$ term.

\begin{tabular}{ccccc}
\hline$j$ & $f_{3, j}\left(\mathrm{~d}^{-1}\right)$ & $P_{3, j}(\mathrm{~d})$ & $A_{3, j}(\mathrm{mmag})$ & $\Phi_{3, j}(\mathrm{rad})$ \\
\hline 1 & $5.50257795 \pm 0.00000033$ & $0.181733000 \pm 0.000000011$ & $7.97 \pm 0.10$ & $1.914 \pm 0.013$ \\
2 & $5.50405957 \pm 0.00000058$ & $0.181684080 \pm 0.000000019$ & $4.24 \pm 0.10$ & $2.484 \pm 0.024$ \\
3 & $5.50396544 \pm 0.00000127$ & $0.181687187 \pm 0.000000042$ & $1.84 \pm 0.09$ & $2.348 \pm 0.049$ \\
\hline
\end{tabular}

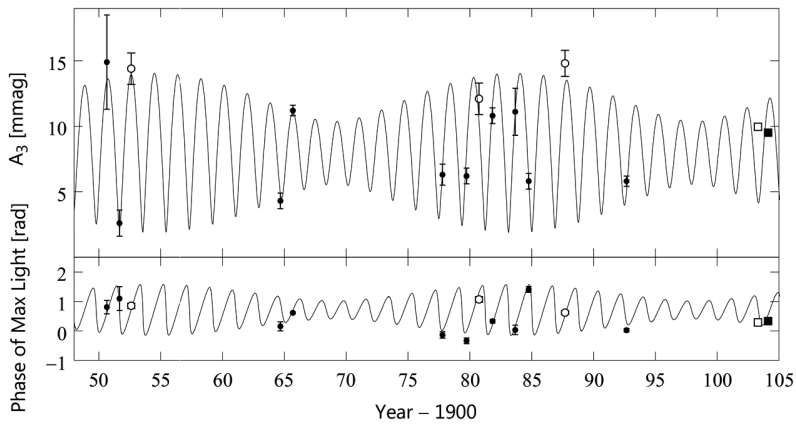

Figure 16. Upper panel: the amplitude of the $f_{3}$ term, $A_{3}$, computed from the parameters of the three-frequency fit of Table 5 (solid line), compared with the yearly mean blue-light $1950-1992 A_{3}$ from table A1 of JP96 and table 1 of JP99 (the dots and circles; the latter represent data of lower weight) and the mean $2003 y$ and $v A_{3}$ (the filled and empty square, respectively). The 2003 error bars are not plotted because they would be of about the same size as the symbols. Lower panel: the same for the phase of maximum light of the $f_{3}$ term. In both panels, the 2003 symbols were shifted along the abscissa by \pm 0.5 yr to avoid overlap.

equal to 5.5025928 and $5.5040765 \mathrm{~d}^{-1}$, were subsequently derived by $\mathrm{L} 01$ from RV data. A frequency analysis of $B \& y-12$ showed this term to be a triplet. Using the frequencies read off the amplitude spectra as starting values in a three-frequency nonlinear least-squares fit of equation (1) to $B \& y-12$ resulted in the frequencies, amplitudes and phases listed in Table 6 . The standard deviation of the fit amounted to $5.4 \mathrm{mmag}$. The epochs of observations were reckoned from HJD 244 5784. A comparison of the amplitudes and phases of maximum light computed from the parameters listed in Table 6 with observations is shown in Figs 16 and 17. The observed and computed phases of maximum light were obtained from the observed and computed maxima using equation (5) with $f=f_{3,1}$ from Table 6.

The new values of $f_{3,1}$ and $f_{3,2}$ are very nearly equal to those obtained by JP99. The new value of the beat-period is equal to $674.94 \pm 0.30 \mathrm{~d}$, in good agreement with the beat-period between the L01's periods $P_{3}$ and $P_{3}^{+}$, mentioned in the Introduction. The third frequency, placed asymmetrically between $f_{3,1}$ and $f_{3,2}$, gives rise to two beat-periods, $720.73 \pm 0.35 \mathrm{~d}$ and $29.09 \pm 0.43 \mathrm{yr}$.

\section{SUMMARY AND DISCUSSION}

Over the 179.2-d interval spanned by the present multisite $u v y$ data, the amplitude of the $f_{1}$ term was constant but the amplitudes of the $f_{2}$ and $f_{3}$ terms, $A_{2}$ and $A_{3}$, increased by several mmags (see Figs 2 and 3). The latter fact made the usual procedure of pre-whitening inapplicable. In Section 3, using the values of the three frequencies determined from all available $B, b$ and $y$ data (Section 7), we pre-whitened the data piecewise, in segments so long that the three terms could be resolved but short enough to neglect the variation of $A_{2}$ and $A_{3}$. In the pre-whitened data, we detected two low-amplitude terms having frequencies $f_{5}=11.3582$ and $f_{4}=6.2990 \mathrm{~d}^{-1}$. The former frequency is equal to $f_{2}+f_{3}$, the latter is new. The am-
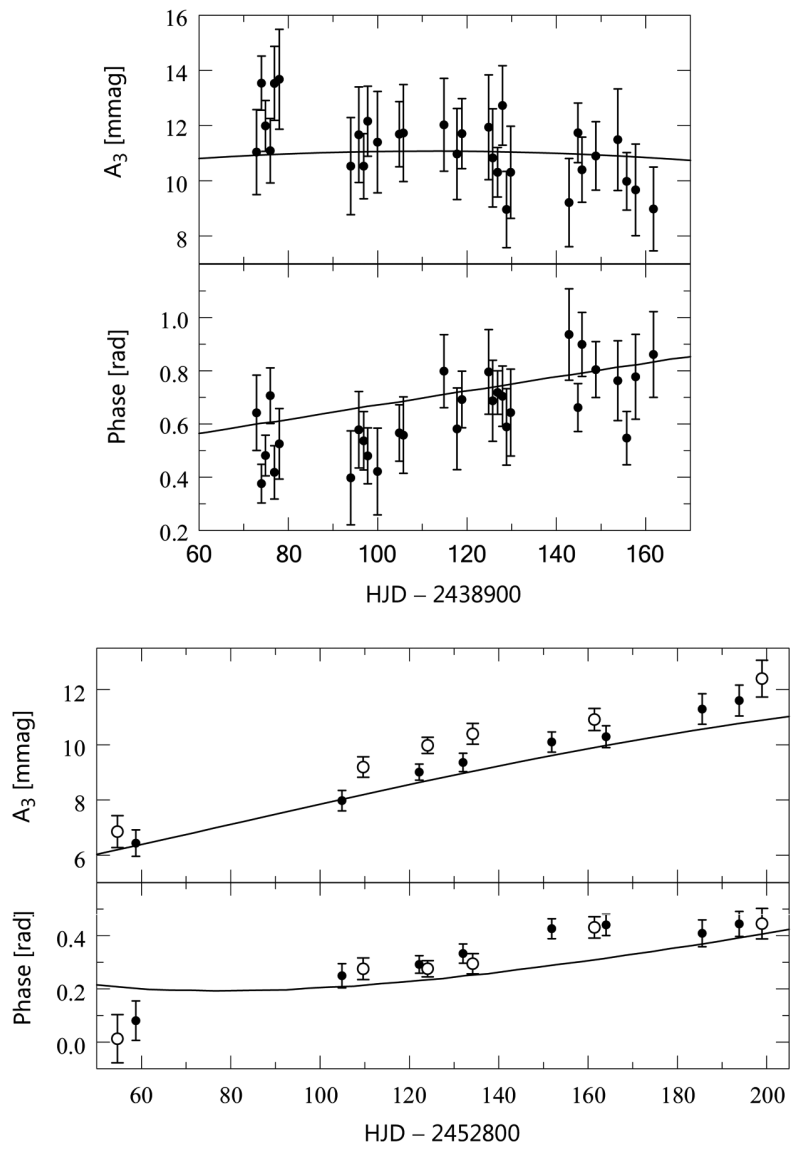

Figure 17. Upper half, upper panel: the amplitude of the $f_{3}$ term, $A_{3}$, computed from the parameters of the three-frequency fit of Table 6 (solid line), compared with the nightly $1965 B$-filter $A_{3}$ from JP96 (dots). Lower half, upper panel: The same for the $y$ and $v A_{3}$ derived in Section 3 (the dots and circles, respectively). Upper half, lower panel: the phase of maximum light of the $f_{3}$ term, computed from the parameters of the three-frequency fit of Table 6 (solid line), compared with the $1965 B$-filter phases of maximum light (dots). Lower half, lower panel: the same for the $y$ and $v$ phases of maximum light (the dots and circles, respectively).

plitudes of the $f_{5}$ term amount to $0.57 \pm 0.09,0.64 \pm 0.10$ and $0.92 \pm 0.15 \mathrm{mmag}$ for $y, v$ and $u$, respectively, while those of $f_{4}$, to $0.64 \pm 0.09,0.61 \pm 0.10$ and $1.05 \pm 0.15 \mathrm{mmag}$, respectively. The frequencies $2 f_{\text {orb }}, 2 f_{1}, f_{1}+f_{3}$ and $f_{1}+f_{2}$ seen in 1965 (see the Introduction) were not found. Why $f_{2}+f_{3}$ was present in 1965 and 2003-2004 while $2 f_{1}, f_{1}+f_{3}$ and $f_{1}+f_{2}$ were absent in 2003-2004 is easy to understand: in 2003-2004 $A_{2}$ was moderately smaller and $A_{3}$ was slightly smaller than in 1965 (see Figs 14 and 16) while $A_{1}$ decreased between 1965 and 2003-2004 to about one fourth of its 1965 value (see Fig. 12). The fact that $2 f_{\text {orb }}$ was missing in 2003-2004 suggests that it may be related to $f_{1}$. Finally, $f_{5}$ was not detected in 1965 because its $V$ and $B$ amplitudes were then below $\sim 0.40 \mathrm{mmag}$. 
With the $f_{i}(i=1, \ldots, 5)$ terms taken into account, we repeated the piecewise pre-whitening. The resulting residuals were used in Section 4 to plot the eclipse light curves (Figs 5 and 6). The light curves show no ellipticity effect and no secondary eclipse can be detected. However, a marginal reflection effect is present. From the $y$ light-curves and the L01 spectroscopic elements $\omega$ and $e$, we computed the relative radius of the primary, $r_{\mathrm{p}}$, the orbital inclination, $i$, and the reflected light from the secondary, $S_{\mathrm{s}}$, for a range of $k=r_{\mathrm{s}} / r_{\mathrm{p}}$ by means of EBOP (Etzel 1981). This allowed an examination of the position of the secondary component in the HR diagram in relation to pre-MS evolutionary tracks (Fig. 7), leading to the conclusions that (1) the secondary component is in the pre-MS phase of its evolution, and (2) the parameters of the system can be constrained to $0.21 \lesssim k \lesssim 0.29,0.125 \lesssim r_{\mathrm{p}} \lesssim 0.132$ and $83.4 \gtrsim i \gtrsim 82.0$.

For $f_{1}, f_{2}$ and $f_{3}$, the $u v y$ and the Geneva $U B G$ amplitude ratios are derived from the multisite data and compared with the theoretical ones for the spherical-harmonic degree $\ell=0, \ldots, 4$ in Section 6. The theoretical amplitude ratios were computed using $T_{\text {eff }}$ and $\log g$ of 16 Lac obtained in Section 5. In Section 6, the highest degree, $\ell=4$, is shown to be incompatible with the observations. The first term, $f_{1}$, could be identified with an $\ell=0$ mode, while the third, $f_{3}$, with an $\ell=1$ mode. In the case of $f_{2}$, an unambiguous sphericalharmonic degree identification was not possible: it can be either an $\ell=2$ or 3 mode, with the latter possibility less likely because of the effect of cancellation in integrating over the stellar disc.

In Section 7, using the present $y$-filter magnitudes and archival blue-filter magnitudes, we investigate the long-term variation of the amplitudes and phases of the three high-amplitude terms over the interval of $53.4 \mathrm{yr}$ spanned by the data. In the case of $f_{1}$, the magnitudes can be represented by means of a sum of five sinusoidal components with closely spaced frequencies (see Table 4). The first three frequencies form an equally spaced triplet with a spacing of $0.0137 \mathrm{yr}^{-1}$, implying a time-scale of $73 \mathrm{yr}$, in agreement with JP99 and L01. The sum of the five components accounts very well for the non-sinusoidal shape of the variation seen in Fig. 12. Since the $f_{1}$ mode is radial, the regularities in the frequency spacings suggest an underlying amplitude and phase modulation of a single pulsation mode.

In the case of $f_{2}$, we could represent the 1950-2003 magnitudes by a sum of four sinusoidal components with uniformly variable amplitudes (see Table 5). Of these, three components $\left(f_{2,2}, f_{2,1}\right.$ and $f_{2,4}$ in the order of increasing frequency) form a triplet very nearly equidistant in frequency with a mean separation of $0.002628 \mathrm{~d}^{-1}$, corresponding to a beat period of $380.5 \mathrm{~d}$, while the $f_{2,3}$ component precedes the first member of the triplet by $0.023 \mathrm{yr}^{-1}$, corresponding to a beat-period of $43 \mathrm{yr}$. The 380.5-d beat-period dominates the variation of the amplitude and phase (see Fig. 14); the 43-yr beat-period is close to the time-scale of the variation of $A_{2}$ derived by JP99 from the $1950-1992$ data. The $f_{2,2}, f_{2,1}, f_{2,4}$ triplet may be the result of (1) rotational splitting of a non-radial mode, (2) an accidental coincidence of two non-radial modes, one rotationally split, and (3) an accidental coincidence of three non-radial modes. In all cases, only some members of the rotationally split multiplets would be excited to observable amplitudes. In (1) and (2), the velocity of the star's rotation can be computed from the Ledoux first-order formula using the radius from the eclipse solutions. The results are $V_{\text {rot }} \lesssim 1 \mathrm{~km} \mathrm{~s}^{-1}$ if (1), and $V_{\text {rot }} \lesssim 2 \mathrm{~km} \mathrm{~s}^{-1}$ if (2). These numbers are in severe conflict with the observed $V_{\text {rot }} \sin i$ (Głębocki et al. 2000). Thus, we are left with (3) and the problem why three accidentally coincident modes should be nearly equidistant in frequency. Finally, there is the possibility that our representation by means of a sum of four sinusoidal components may be merely a formal description of the complex long-term variation of the amplitude and phase of the $f_{2}$ term.

JP96 have noted that the reciprocal of the growth rates of several $\ell \leq 2$ pulsation modes which are excited in models of $16 \mathrm{Lac}$ and can be identified with the $f_{1}$ and $f_{2}$ terms are of the same order of magnitude as the time-scales of 73 and 43 yr just mentioned. Since amplitude and phase modulation on a time-scale of the order of the reciprocal of the growth rate is predicted by the theory of nonlinear interaction of pulsation modes, JP96 suggested that these time-scales in the observed long-term behaviour of the $f_{1}$ and $f_{2}$ modes result from (1) the 1:1 resonance between them, or (2) a resonant coupling to other modes. These points still await theoretical verification.

Finally, the $f_{3}$ 1950-2003 magnitudes could be represented by a sum of three sinusoidal components (see Table 6). The first two components have frequencies $f_{3,1}$ and $f_{3,2}$ very nearly equal to those derived by JP96 and JP99 from the data available at the time. These two frequencies produce a beat period of $720.73 \pm 0.35 \mathrm{~d}$ which dominates the amplitude and phase variation (see Fig. 16). The frequencies $f_{3,3}$ and $f_{3,2}$ produce a beat period of $29.09 \pm 0.43 \mathrm{yr}$. As in the case of $f_{2}$, assuming that the $f_{3,1}, f_{3,2}$ doublet is due to rotational splitting of a non-radial mode leads to $V_{\text {rot }} \lesssim 1 \mathrm{~km} \mathrm{~s}^{-1}$, much smaller than observed. JP96 suggested that the doublet represents an accidental near-coincidence of two self-excited modes. In view of the unambiguous identification of the harmonic degree of the $f_{3}$ term (Section 6), these two modes must both have $\ell=1$. On the other hand, the members of the $f_{2,2}, f_{2,1}, f_{2,4}$ triplet may have different $\ell$, a situation encountered earlier in 1 Mon (Balona \& Stobie 1980) and 12 Lac (Handler et al. 2006).

\section{ACKNOWLEDGEMENTS}

We are indebted to Dr Paul B. Etzel for providing the source code of his computer program EBOP and explanations. GH was supported by the Polish NCN grant 2011/01/B/ST9/05448. AP, ZK and GM acknowledge the support from the NCN grant 2011/03/B/ST9/02667. In this research, we have used the Aladin service, operated at CDS, Strasbourg, France, and the SAO/NASA Astrophysics Data System Abstract Service.

\section{REFERENCES}

Aerts C., Lehmann H., Scuflaire R., Dupret M. A., Briquet M., De Ridder J., Thoul A., 2003a, in Thompson M. J., Cunha M. S., Monteiro M. J. P. F. G., eds, Asteroseismology Across the HR Diagram. Kluwer, Dordrecht, p. 493

Aerts C., Lehmann H., Briquet M., Scuflaire R., Dupret M. A., De Ridder J., Thoul A., 2003b, A\&A, 399, 639

Asplund M., Grevesse N., Sauval A. J., Allende Prieto C., Kiselman D., 2004, A\&A, 417, 751

Asplund M., Grevesse N., Sauval A. J., Scott P., 2009, ARA\&A, 47, 481

Balona L. A., 1994, MNRAS, 268, 119

Balona L. A., Evers E. A., 1999, MNRAS, 302, 349

Balona L. A., Stobie R. S., 1980, MNRAS, 190, 931

Bessell M. S., 2000, PASP, 112, 961

Blaauw A., 1964, ARA\&A, 2, 213

Breger M. et al., 1993, A\&A, 271, 482

Burnham S. W., 1894, Publ. Lick Obs., 2, 53

Chen B., Vergely J. L., Valette B., Carraro G., 1998, A\&A, 336, 137

Code A. D., Davis J., Bless R. C., Brown R. H., 1976, ApJ, 203, 417

Crawford D. L., 1978, AJ, 83, 48

Daszyńska-Daszkiewicz J., Dziembowski W. A., Pamyatnykh A. A., Goupil M.-J., 2002, A\&A, 392, 151

Davis J., Shobbrook R. R., 1977, MNRAS, 178, 651

Dziembowski W. A., 1977, Acta Astron., 27, 95 
Dziembowski W. A., Jerzykiewicz M., 1996, A\&A, 306, 436

Etzel P. B., 1981, in Carling E. B., Kopal Z., eds, Photometric and Spectroscopic Binary Systems. Reidel, Dordrecht, p. 111

Fabricius C., Makarov V. V., 2000, A\&A, 356, 141

Ferguson J. W., Alexander D. R., Allard F., 2005, ApJ, 623, 585

Fitch W. S., 1969, ApJ, 158, 269

Flower P. J., 1996, ApJ, 469, 355

Głębocki R., Gnaciński P., Stawikowski A., 2000, Acta Astron., 50, 509

Handler G. et al., 2000, MNRAS, 318, 511

Handler G. et al., 2006, MNRAS, 365, 327

Hauck B., Mermilliod M., 1998, A\&AS, 129, 431

Heynderickx D., 1994, A\&A, 283, 835

Iglesias C. A., Rogers F. J., 1996, ApJ, 464, 943

Jarzębowski T., Jerzykiewicz M., Le Contel J.-M., Musielok B., 1979, Acta Astron., 29, 517

Jerzykiewicz M., 1980, in Hill H. A., Dziembowski W. A., eds, Non-radial and Non-linear Stellar Pulsation. Springer, Berlin p. 125

Jerzykiewicz M., 1993, Acta Astron., 43, 13

Jerzykiewicz M., 1994, in Balona L. A., Henrichs H. F., Le Contel J.-M., eds, Proc. IAU Symp. 162, Pulsation, Rotation, and Mass-Loss in Early-Type Stars. Kluwer, Dordrecht, p. 3

Jerzykiewicz M., Pigulski A., 1996, MNRAS, 282, 853

Jerzykiewicz M., Pigulski A., 1999, MNRAS, 310, 804

Jerzykiewicz M., Handler G., Shobbrook R. R., Pigulski A., Medupe R., Mokgwetsi T., Tlhagwane P., Rodríguez E., 2005, MNRAS, 360, 619

Kurucz R. L., 1994, CD-ROM 19

Kurucz R. L., 2004, Available at: (http://kurucz.harvard.edu)

Lee O. J., 1910, ApJ, 32, 307

Lehmann H. et al., 2001, A\&A, 367, 236

Mermilliod M., 1991, Catalogue of Homogeneous Means in the UBV System. Institut d'Astronomie, Univ. Lausanne

Montgomery M. H., O’Donoghue D., 1999, Delta Scuti Star Newsl., 13, 28

Moon T. T., Dworetsky M. M., 1985, MNRAS, 217, 305

Morris S. L., 1985, ApJ, 295, 143

Napiwotzki R., Schönberner D., Wenske V., 1993, A\&A, 268, 653

Nelson B., Davis W. D., 1972, ApJ, 174, 617

Niemczura E., Daszyńska-Daszkiewicz J., 2005, A\&A, 433, 659

Nieva M.-F., Przybilla N., 2012, A\&A, 539, A143

Pamyatnykh A. A., Dziembowski W. A., Handler G., Pikall H., 1998, A\&A, 333,141

Pigulski A., Jerzykiewicz M., 1988, Acta Astron., 38, 401

Popper D. M., Etzel P. B., 1981, AJ, 86, 102

Prugniel Ph., Vauglin I., Koleva M., 2011, A\&A, 531, A165

Rica Romero F. M., 2010, Rev. Mex. Astron. Astrofis., 46, 263

Rodríguez E., López-González M. J., López de Coca P., 2000, A\&AS, 144, 369

Rogers F. J., Nayfonov A., 2002, ApJ, 576, 1064

Royer F., Zorec J., Gomez A. E., 2007, A\&A, 463, 671

Russell H. N., Merrill J. E., 1952, Contrib. Princeton Univ. Obs., No. 26

Sareyan J.-P., Chauville J., Chapellier E., Alvarez M., 1997, A\&A, 321, 145

Seaton M. J., 2005, MNRAS, 362, L1

Serenelli A. M., Basu S., Ferguson J. W., Asplund M., 2009, ApJ, 705, 123

Sterken C., Jerzykiewicz M., 1993, Space Sci. Rev., 62, 95

Stetson P. B., 1987, PASP, 99, 191

Struve O., Bobrovnikoff N. T., 1925, ApJ, 62, 139

Thoul A. et al., 2003, A\&A, 406, 287

Tognelli E., Prada Moroni P. G., Degl'Innocenti S., 2011, A\&A, 533, 109

Van Hamme W., 1993, AJ, 106, 2096

van Leeuwen F., 2007, A\&A, 474, 653

\section{APPENDIX A: THE VARIABLE COMPARISON STAR 2 ANDROMEDAE}

\section{A1 Frequency analysis}

As mentioned in the Introduction, the comparison star 2 And turned out to be a small-amplitude variable. Fig. A1 shows the results of a

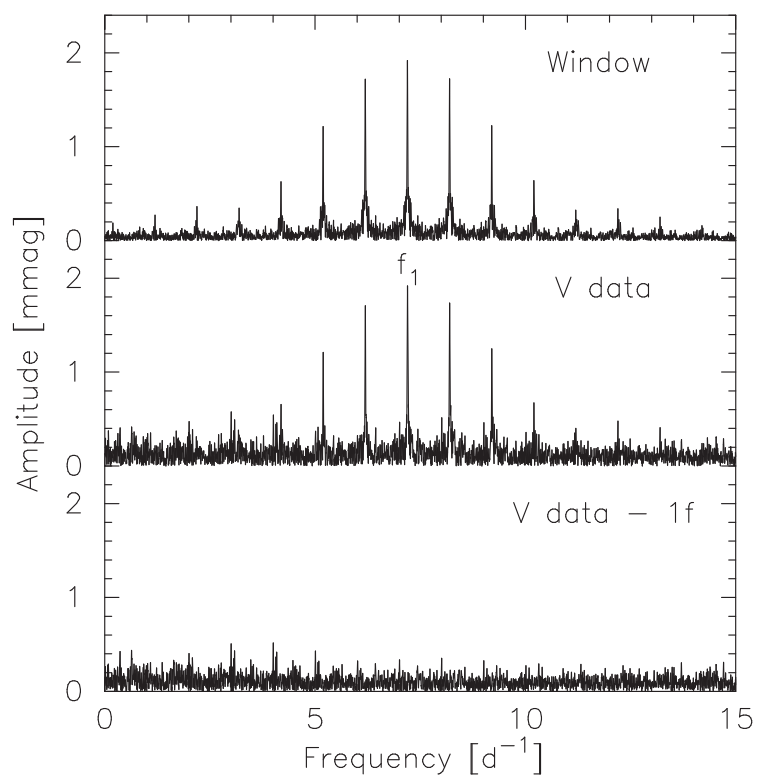

Figure A1. Amplitude spectrum of 2 And. The top panel shows the spectral window of the data, followed by the periodogram of the data in the middle panel. Pre-whitening with $f_{1}$ leaves only noise in the residuals (bottom panel), aside from some residual extinction effects (peaks at 2.0, 3.0, 4.0 and $\left.5.0 \mathrm{~d}^{-1}\right)$.

Table A1. Amplitudes and relative phases of the single frequency $7.195301 \mathrm{~d}^{-1}$ for the variable comparison star 2 And. Formal error estimates (following Montgomery \& O'Donoghue 1999) for the amplitudes and phases are given.

\begin{tabular}{lcr}
\hline Filter & $\begin{array}{c}\text { Amplitude } \\
(\mathrm{mmag})\end{array}$ & \multicolumn{1}{c}{$\begin{array}{c}\phi_{x}-\phi_{y} \\
(\mathrm{rad})\end{array}$} \\
\hline$u$ & $1.72 \pm 0.13$ & $-0.16 \pm 0.09$ \\
$v$ & $1.13 \pm 0.08$ & $-0.05 \pm 0.08$ \\
$y$ & $1.91 \pm 0.07$ & 0 by definition \\
$U$ & $2.07 \pm 0.29$ & $0.07 \pm 0.14$ \\
$B 1$ & $1.57 \pm 0.25$ & $-0.17 \pm 0.16$ \\
$B$ & $1.69 \pm 0.25$ & $-0.06 \pm 0.15$ \\
$B 2$ & $1.65 \pm 0.26$ & $-0.22 \pm 0.16$ \\
$V 1$ & $2.26 \pm 0.25$ & $-0.02 \pm 0.12$ \\
$V$ & $2.05 \pm 0.22$ & $0.01 \pm 0.12$ \\
$G$ & $2.42 \pm 0.26$ & $-0.04 \pm 0.11$ \\
\hline
\end{tabular}

frequency analysis of the differential $y$ magnitudes 2 And - 10 Lac. The less accurate Sierra Nevada and Piszkéstetô observations were omitted.

The variability of 2 And can be described by a single frequency (Fig. A1), which is confirmed by the analysis of the measurements in the other filters. Non-linear least-squares fitting to our $y$-filter data gives a value of $7.195301 \pm 0.00012 \mathrm{~d}^{-1}$, whereby the error estimate was computed with the formulae of Montgomery \& O'Donoghue (1999). Fitting a sine curve of this frequency to the data results in the amplitudes and relative phases, $\phi_{x}-\phi_{y}(x=u$, $v, U, B 1, B, B 2, V 1, V, G)$, listed in Table A1.

\section{A2 The cause of variability of 2 And}

Given the spectral type of A3 Vn and the period of the variability, one would immediately suspect that the light variations of 2 And are due to $\delta$ Scuti-type pulsation. In fact, the star was so classified 


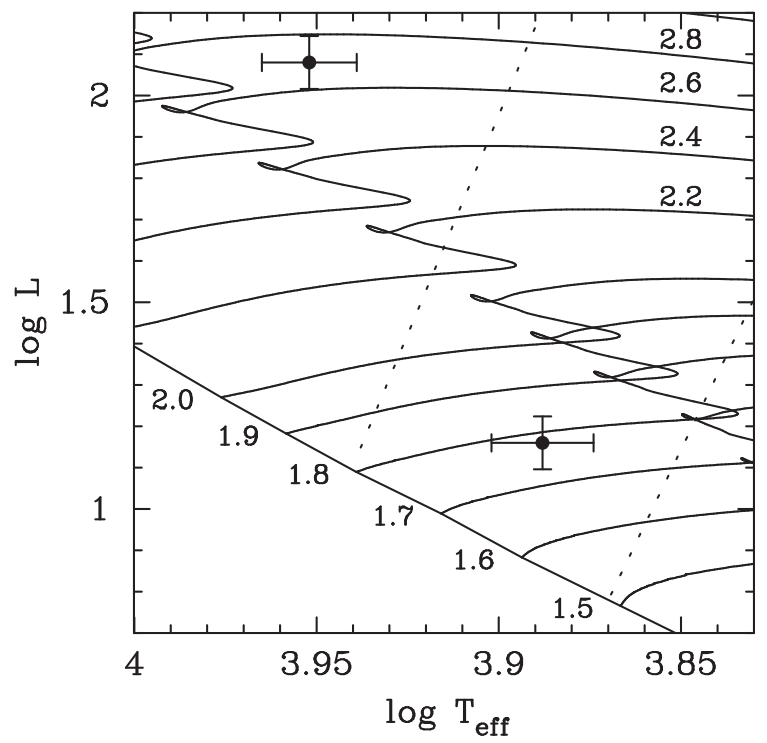

Figure A2. The positions of 2 And A and B in the theoretical HR diagram. Some stellar model evolutionary tracks labelled with their masses in solar units (solid lines) are included. The borders of the $\delta$ Scuti instability strip (adopted from Rodríguez, López-González \& López de Coca 2000, dotted lines) are included for comparison. The solid diagonal line is the ZAMS.

by Handler et al. (2006). However, if 2 And were a $\delta$ Scuti variable, the amplitudes are generally expected to increase from $y$ to $v$ and from $G$ to $B 1$, and then level out or drop again towards $u$ and $U$ (e.g. see Heynderickx 1994). Interestingly, just the inverse is the case. In the following, we investigate possible causes for this observation.

2 And is not a single star. It is a close visual binary discovered over a century ago (Burnham 1894). The two components are physically associated. The latest determination of the orbital period is $74 \mathrm{yr}$ with an eccentricity of 0.8 and a semimajor axis of 0.23 arcsec (Rica Romero 2010). Transforming the Tycho-2 photometry of the two components (Fabricius \& Makarov 2000), to the standard Johnson system according to Bessell (2000) gives: $V_{\mathrm{A}}=5.24 \mathrm{mag},(B-V)_{\mathrm{A}}=0.07 \mathrm{mag}$ for 2 And $\mathrm{A}$, and $V_{\mathrm{B}}=7.51 \mathrm{mag},(B-V)_{\mathrm{B}}=0.23 \mathrm{mag}$ for 2 And B. Therefore $V_{\mathrm{A}+\mathrm{B}}=5.113 \mathrm{mag}$ and $(B-V)_{\mathrm{A}+\mathrm{B}}=0.086 \mathrm{mag}$, in reasonable agreement with the measured $V=5.100 \mathrm{mag}$ and $B-V=0.094 \mathrm{mag}$ for the system (Mermilliod 1991).

The revised Hipparcos parallax of 2 And (van Leeuwen 2007) implies a distance of $129 \pm 9$ pc. Concerning reddening, a comparison of the results from the Galactic reddening law of Chen et al. (1998) for 2 And, and the reddening of two stars within $4^{\circ}$ of 2 And in the sky, at a similar Hipparcos distance (HR 8870 and HD 218394) lead us to adopt $E(B-V)=0.022 \pm 0.022 \mathrm{mag}$ and $A(V)=0.07 \pm 0.07 \mathrm{mag}$.

We then arrive at $M_{\mathrm{V}}=-0.39 \pm 0.16 \mathrm{mag}$ for 2 And $\mathrm{A}$ and $1.88 \pm 0.16 \mathrm{mag}$ for 2 And B. The relations of Flower (1996) yield estimates of $T_{\text {eff }}=8950 \pm 250 \mathrm{~K}$ and $M_{\text {bol }}=-0.45 \pm 0.16 \mathrm{mag}$ for 2 And $\mathrm{A}$, and $7720 \pm 250 \mathrm{~K}$ and $1.85 \pm 0.16 \mathrm{mag}$ for 2 And B. The positions of the two components are shown in a theoretical HR diagram in Fig. A2. The evolutionary tracks plotted in this figure were computed with the Warsaw-New Jersey stellar evolution code, the OPAL equation of state and the OPAL opacity tables (Iglesias \& Rogers 1996), a hydrogen abundance of $X=0.7$, a metal abundance of $Z=0.012$ (Asplund et al. 2004), and no convective core overshooting. We assumed $V_{\text {rot }}=250 \mathrm{~km} \mathrm{~s}^{-1}$ on the zero-age main sequence, so that the observed $V_{\text {rot }} \sin i=212 \mathrm{~km} \mathrm{~s}^{-1}$ (Royer, Zorec \& Gomez 2007) would be approximately matched.

One finds that 2 And $\mathrm{A}$ is an $M=2.7 \pm 0.1 \mathrm{M}_{\odot}$ star that probably has already left the main sequence $(\log g=3.40 \pm 0.12$, log age $=8.57 \pm 0.04)$. Including core overshooting would decrease $M$ and the age but $\log g$ would remain virtually unchanged. 2 And A is located off the $\delta$ Scuti instability strip. 2 And B is situated in the centre of the strip $\left(M=1.78 \pm 0.06 \mathrm{M}_{\odot}, \log g=3.90 \pm 0.16\right.$, log age $=8.84_{-0.18}^{+0.07}$ ).

With these parameters in hand, we shall test four possible hypotheses to explain the cause of the variability of 2 And: $\delta$ Scuti pulsation or ellipsoidal variability, of either 2 And A or 2 And B. Rotational modulation can be excluded immediately because either star would have to rotate faster than its breakup velocity.

Regarding the hypothesis of $\delta$ Scuti pulsation, we computed theoretical pulsation amplitudes of $\delta$ Scuti models for the Strömgren and Geneva passbands in the parameter ranges depicted in Fig. A2 for an assumed RV amplitude, using the methodology by Balona \& Evers (1999). We note that whilst computing realistic absolute amplitudes is still out of reach, amplitude ratios are easily obtainable.

We computed theoretical pulsation amplitudes of $\delta$ Scuti models for the Strömgren and Geneva passbands. From the flux ratio of 2 And $\mathrm{A}$ to 2 And $\mathrm{B}$ in the $y$ band (estimated from $V_{\mathrm{A}}$ and $V_{\mathrm{B}}$ ) and the observed $y$ amplitude from Table A1 we then determined the undiluted photometric amplitudes of 2 And A and 2 And B in the Strömgren $y$ band, ( $A_{\text {true }}=0.0022$ and $0.0215 \mathrm{mag}$, respectively), and used the model amplitudes to predict the intrinsic amplitudes in the other bands. As the next step, we determined the flux ratio of the two components of 2 And in the different passbands. To this end, we used Kurucz (1994) model atmospheres, representing 2 And A with a $T_{\text {eff }}=9000 \mathrm{~K}, \log g=3.5$ model atmosphere, and 2 And B with a $T_{\text {eff }}=7750 \mathrm{~K}, \log g=4.0$ model atmosphere. We then integrated the monochromatic fluxes from these model atmospheres over the 10 photometric passbands used, and scaled the resulting fluxes to the observed ratio in $y$.

The results of these computations can be summarized as follows: the behaviour of the pulsation amplitudes with wavelength is inconsistent with the observations for any kind of assumed pulsation of 2 And A. The reason is that the temperature of the star is so high, that the pulsation amplitudes always increase towards shorter wavelength, no matter which spherical degree of the pulsation was tested (we checked for $0 \leq l \leq 8$ ). The additional flux of 2 And B does not change this behaviour due to the considerable luminosity difference.

Assuming that 2 And B was a $\delta$ Scuti pulsator, and considering the amplitude contamination due to the light of 2 And A, we find that the observed pulsation amplitude versus wavelength dependence is only consistent with modes of even spherical degree of $l \geq 6$. We recall that in such a scenario the intrinsic pulsation amplitude of 2 And B in the Strömgren $y$ band, $A_{\text {true }}$ is 0.0215 mag. Geometric cancellation of modes with $l \geq 6$ decreases the observed amplitudes to less than $1 / 50$ of the intrinsic value (Daszyńska-Daszkiewicz et al. 2002), i.e. the intrinsic pulsation amplitude would be enormous in this case. We therefore consider $\delta$ Scuti pulsation of either of the two A-type stars in the 2 And system as highly unlikely.

A remaining possibility is an ellipsoidal variation of either component. Consequently, we repeated the previous procedure under the assumption of no colour dependence of the amplitude. We found that the resulting wavelength-amplitude dependence explains the observations much better than the $\delta$ Scuti-pulsation hypothesis. This implies an orbital period of twice the observed value. Using the effective temperatures, the luminosities and the masses derived 
above we find from the Kepler's third law that in the case of 2 And $\mathrm{A}$ the sum of the radii of the components $\mathrm{Aa}$ and $\mathrm{Ab}, R_{\mathrm{Aa}}+R_{\mathrm{Ab}}$, would be a factor of about 2 greater than the semimajor axis of the relative orbit, $a$, regardless of whether we assume (1) equal luminosity and mass components, or (2) the secondary component much fainter and less massive than the primary component. In the case of 2 And $\mathrm{B}$, assumption (1) leads to $R_{\mathrm{Ba}}+R_{\mathrm{Bb}} \approx 1.5 a$, while assuming (2) one gets $R_{\mathrm{Ba}} \approx 1.0 a$. Thus, an ellipsoidal variation of 2 And $\mathrm{B}$, arising from a tidal distortion of component $\mathrm{Ba}$ by a much less massive secondary component $\mathrm{Bb}$ in a tight orbit may be the cause of the variability of 2 And.

In order to examine the last possibility let us use the lowest order approximation, certainly adequate in the present case, according to which the amplitude of the light variation due to aspect changes of the tidally distorted primary can be expressed by means of the following formula:

$\delta m=1.629 A_{\lambda} q\left(R_{\mathrm{Ba}} / a\right)^{3} \sin ^{2} i$,

where $\delta m$ is expressed in magnitudes, $A_{\lambda}$ is the photometric distortion parameter of Russell \& Merrill (1952), $q$ is the mass ratio of the components, and $i$ is the inclination of the orbit to the tangent plane of the sky. We note that this formula is equivalent to equation 6 of Morris (1985). Neglecting the light variation of the secondary, we have $\delta m=A_{\text {true }}$. Then, taking into account the fact that in the present case $A_{\lambda}\left(R_{\mathrm{Ba}} / a\right)^{3} \approx 1$, we get $q \approx 0.013$ and 0.052 for $i=90^{\circ}$ and $30^{\circ}$, respectively. The corresponding masses of the secondary component are equal to $\sim 0.03$ and $\sim 0.11 \mathrm{M}_{\odot}$, i.e. they are in the range of the masses of brown dwarfs. Thus, our hypothesis that 2 And B is an ellipsoidal variable leads to the following model: the 2 And $\mathrm{Bb}$ component is a brown dwarf in a tight orbit around 2 And $\mathrm{Ba}$, a late $\mathrm{A}$ or an early $\mathrm{F}$ star. The amplitude of the RV variation, $K_{1} \sin i$, would be equal to 10 and $20 \mathrm{~km} \mathrm{~s}^{-1}$ for $i=90^{\circ}$ and $30^{\circ}$, respectively. According to the ephemeris provided by Rica Romero (2010), the separation of the components A and B will be 0.135 arcsec in 2014 January, decreasing to 0.046 arcsec by 2018 . In the near future, it will be thus impossible to get a spectrogram of 2 And B without an overwhelming contribution from 2 And A. Consequently, measuring the RV of 2 And $\mathrm{B}$ may be very difficult.

This paper has been typeset from a $\mathrm{T}_{\mathrm{E}} \mathrm{X} / \mathrm{L} \mathrm{T} \mathrm{E} \mathrm{X}$ file prepared by the author. 